\title{
Discrete-Time Nonlinear Feedback Control of Multivariable Processes
}

\author{
Masoud Soroush and Costas Kravaris \\ Dept. of Chemical Engineering, The University of Michigan, Ann Arbor, MI 48109
}

\begin{abstract}
Discrete-time nonlinear feedback control laws are derived for multivariable nonlinear processes, whose "delay-free" parts are minimum phase. These include mixed errorand state-feedback, error-feedback, and mixed error- and output-feedback laws, which can induce linear input-output closed-loop response. This study is carried out within the framework of the discrete-time globally linearizing control. The broader class of nonlinear processes, in which controlled outputs as well as some other process variables are measured, are also considered. A mixed error-and output-feedback control law is derived for this class of processes. The conditions under which the mixed error- and output-feedback can be applied to a process operating at or around an open-loop unstable equilibrium point are determined. The application and performance of the derived control laws are illustrated by the numerical simulation of a chemical reactor.
\end{abstract}

\section{Introduction}

Since the mid $1980 \mathrm{~s}$, the area of nonlinear process control has witnessed an exponential growth. The extent of the growth and the current status of the area have been discussed in several recent review articles (McLellan et al., 1990; Bequette, 1991; Biegler and Rawlings, 1991; Kravaris and Arkun, 1991). Among various research directions that have been pursued to study the problem of nonlinear controller synthesis, the most popular directions have been: the geometric or system-theoretic point of view with its foundation on nonlinear systems theory, formulated via differential geometric methods; the model-predictive point of view that follows an optimization approach. Although these two points of view initially appeared as disjointed research directions, intimate connections have been recently reported (Soroush and Kravaris, 1992a,b).

For the nonlinear process, although input-output operators have provided some significant theoretical insights into the problem of nonlinear controller synthesis (Economou, 1985; Li et al., 1990; Daoutidis and Kravaris, 1994; Kravaris et al., 1994), a stand-alone general input-output synthesis approach cannot exist, and any input-output formulation must switch into state space at some point in the synthesis methodology. Because of this, both geometric and modelpredictive approaches have been formulated primarily in a

Correspondence concerning this article should be addressed to M. Soroush, who is currently at the Chemical Engineering Department, Drexel University, Philadelphia, PA 19104. state-space setting. A nonlinear controller synthesis approach, geometric or model-predictive in nature, therefore involves two key steps: synthesis of a state-feedback law (on the basis of desirable closed-loop input-output response characteristics or by minimizing a performance index); development of a state reconstruction technique for the case of incomplete state information. Then the overall control law arises from a combination of the state-feedback and state observer.

While there are a variety of ways to calculate a state-feedback, at the present time there is no general method of nonlinear state-observer design. The available nonlinear stateobserver design methods are either problem dependent or applicable to a very restricted class of nonlinear processes. For example, in the case of a nonlinear process operating only within the domain of attraction of an open-loop stable equilibrium point, one can use a full-order, open-loop observer to reconstruct the states. There are, however, several disadvantages associated with this type of observer: (a) the state estimates are normally very sensitive to model errors and unmeasurable disturbances; and (b) these observers are only applicable to the processes operating only within the domain of attraction of an open-loop stable equilibrium point. To some extent, these deficiencies can be taken care of by using a reduced-order, open-loop observer driven by output measurements. It is an objective of this article to explore the advantages of these reduced-order observers. 
Nonlinear control methods have been formulated both in continuous time in discrete time. The main advantage of the continuous-time formulation is that physical parameters are explicit in the process model and therefore in the control law. The main advantages of discrete-time formulation are that: the process model and control law are directly suitable for computer implementation; the presence of deadtime does not complicate the control problem; and system identification is, in a sense, more straightforward, for example, one can fit the data to a polynomial ARMA model (Hernandez and Arkun, 1993). Because of these appealing features, the discrete-time formulation has been the recent trend in the literature.

The nonlinear control study of this work will be carried out in a state-space synthesis framework and in discrete time. In particular, we employ the notion of input-output linearization and the globally linearizing control (GLC) design methodology (Kravaris and Soroush, 1990; Soroush and Kravaris, 1992b), wherein the control objective is to induce a linear, closed-loop, input-output dynamics with desirable performance characteristics. The present article is a continuation of previous work of the authors and coworkers in designing nonlinear controllers, in the following sense. It provides (a) a multivariable generalization of the results in (Soroush and Kravaris, 1992b) on discrete-time single-input single-output (SISO) nonlinear feedback control, and (b) a discrete-time analog of the continuous-time multivariable results in Kravaris and Soroush (1990) and Daoutidis and Kravaris (1994). In addition to providing a necessary extension of previous results, the present article also proposes a new state-reconstruction method. The broader class of the nonlinear processes, in which controlled outputs as well as some other output variables are measured, are also considered. A mixed error- and output-feedback control law is derived for this class of processes.

This article begins with a brief review of the definitions of relative orders and characteristic matrix. The problem of diagonal time-delay factorization of a class of nonlinear systems is addressed, followed by the concept of minimum-phase behavior. A synthesis formula for input-output linearizing state feedbacks is then derived. Within the framework of the discrete-time GLC, mixed error- and state-feedback, errorfeedback, and mixed error- and output-feedback control laws are derived. The stability of the closed-loop system under each derived control law is studied and the corresponding conditions for closed-loop stability are given. The closed-loop response as well as the control laws are simplified in a number of cases by choosing special closed-loop responses. Finally, the application and performance of the derived control laws are illustrated by a reactor example.

\section{Scope and Mathematical Preliminaries}

This work considers nonlinear, multiinput multioutput (MIMO) systems with equal number of inputs and outputs, described by a discrete-time state-space model of the form

$$
\left\{\begin{aligned}
x(k+1) & =\Phi[x(k), u(k)] \\
y_{i}(k) & =h_{i}[x(k)], \quad i=1, \ldots, m,
\end{aligned}\right.
$$

where $x=\left[x_{1} \cdots x_{n}\right]^{T}, u=\left[u_{1} \cdots u_{m}\right]^{T}$, and $y=\left[y_{1} \cdots y_{m}\right]^{T}$ de- note the vectors of state variables, manipulated inputs, and controlled outputs, respectively, all in the form of deviation variables. It is assumed that $x \in X \subset \mathbb{R}^{n}$ and $u \in U \subset \mathbb{R}^{m}$, where $X$ and $U$ are connected open sets that contain the origin (i.e., the nominal equilibrium point); $\Phi(x, u)$ is an analytic vector function on $X \times U$; and $h(x)=\left[h_{1}(x) \cdots h_{m}(x)\right]^{T}$ is an analytic vector function on $X$.

The model described by Eq. 1 can be viewed as the sampled-data representation of a continuous-time process model of the form

$$
\left\{\begin{array}{l}
\dot{\bar{x}}(t)=f[\bar{x}(t)]+\sum_{j=1}^{m} g_{j}[\bar{x}(t)] \bar{u}_{j}(t) \\
\bar{y}_{i}(t)=h_{i}[\bar{x}(t)], \quad i=1, \ldots, m,
\end{array}\right.
$$

that is, $x(k+1)=\Phi(x(k), u(k))$ equals the solution at time $(k+1) \Delta t$ of the differential equations of Eq. 2 starting at time $k \Delta t$ in $\bar{x}(k \Delta t)=x(k)$ and with a constant $\bar{u}(t)=u(k)$, where $\Delta t$ is the sampling period.

In regard to the general form of Eq. 1, the following points should be made:

- If time-delays are introduced in the inputs and/or outputs of the delay-free system of Eq. 2, the corresponding sampled-data representation of the continuous-time model with time delays will still be in the general form of Eq. 1 (addition of time delay to a system of the form of Eq. 1 simply increases the dimension of the system).

- Unlike the continuous-time model of Eq. 2, the process description of Eq. 1 is not affine in $u$. Consideration of a more general class of models in discrete time is necessary, because (a) the sampled-data representation of a process described by the continuous-time model of Eq. 2 is, in general, not affine in $u$, and (b) even when a discrete-time model is affine in $u$, the input-output linearizing state feedback will be, in general, not affine in the external input.

In what follows, system-theoretic notions for MIMO discrete-time systems will be reviewed briefly. Refer to Nijmeijer and van der Schaft (1990) for details.

Definition 1. For a system of the form of Eq. 1, the relative order of the output $y_{i}$ with respect to the manipulated input vector $u$ is the smallest integer $r_{i}$ for which

$$
\left[\frac{\partial h_{i}(x)}{\partial x}\right]\left[\frac{\partial \Phi(x, u)}{\partial x}\right]^{r_{i}-1}\left[\frac{\partial \Phi(x, u)}{\partial u}\right] \not \equiv[0 \cdots 0]
$$

If such an integer does not exist, we say that $r_{i}=\infty$.

Definition 1 implies that if the relative order $r_{i}=1$, then $\partial\left(h_{i} \circ \Phi\right) / \partial u \not \equiv[0 \cdots 0]$, where " $:$ " denotes composition of functions, that is, $h_{i} \circ \Phi \triangleq h_{i}(\Phi(x, u))$. If the relative order $r_{i}=2$, then $\partial\left(h_{i} \circ \Phi\right) / \partial u \equiv[0 \cdots 0],\left(h_{i} \circ \Phi\right.$ is a function of $x$ only), but $\partial\left(h_{i} \circ \Phi \circ \Phi\right) / \partial u \neq[0 \cdots 0]$. If the relative order $r_{i}=$ 3 , then $\partial\left(h_{i} \circ \Phi\right) / \partial u \equiv[0 \cdots 0]$ and $\partial\left(h_{i} \circ \Phi \circ \Phi\right) / \partial u \equiv[0 \cdots 0]$, (both $h_{i} \circ \Phi$ and $h_{i} \circ \Phi \circ \Phi$ are functions of $x$ only), but $\partial\left(h_{i} \circ \Phi \circ \Phi \circ \Phi\right) / \partial u \not \equiv[0 \cdots 0]$. Therefore, in the general case, the relative order $r_{i}$ is the smallest integer for which 


$$
h_{i} \circ \underbrace{\Phi \circ \cdots \circ \Phi}_{r_{i} \text { times }}
$$

depends on $a u_{j}$.

In view of the definition and properties of the relative orders $r_{1}, \ldots, r_{m}$, the following notation, which will be used in the subsequent sections, is defined:

$$
\begin{aligned}
& h_{i}^{0}(x) \triangleq h_{i}(x) \\
& h_{i}^{\ell}(x) \triangleq h_{i}^{\ell-1}[\Phi(x, u)], \quad \ell=1, \ldots, r_{i}-1
\end{aligned}
$$

In this notation,

$$
\frac{\partial}{\partial u} h_{i}^{r_{i}-1}[\Phi(x, u)] \neq[0 \cdots 0],
$$

and the following relations hold:

$$
\left\{\begin{array}{l}
y_{i}(k+\ell)=h_{i}^{\ell}[x(k)], \quad \ell=0, \ldots, r_{i}-1 . \\
y_{i}\left(k+r_{i}\right)=h_{i}^{r_{i}-1}\{\Phi[x(k), u(k)]\}
\end{array}\right.
$$

Consequently, the relative order $r_{i}$ is the smallest number of sampling periods after which a manipulated input move $u_{j}(k)$ affects the output $y_{i}$.

If a system output $y_{i}$ does not have a finite relative order $\left(r_{i}=\infty\right)$, this means that none of the manipulated inputs $u_{1}$, $\ldots, u_{m}$ affect the output $y_{i}\left(y_{i}\right.$ is not controllable). In every well-formulated control problem, all the outputs $y_{1}, \ldots, y_{m}$ must possess finite relative orders. Throughout this article, it will be assumed that all the relative orders are finite and $\partial\left[h_{i}^{r_{i}-1}(\Phi(x, u))\right] / \partial u \neq[0 \cdots 0]$ on $X \times U, i=1, \ldots, m$.

In analogy to linear systems, it is straightforward to show that the exact sampled-data representation of a deadtimefree, MIMO, continuous-time system with finite relative orders $\bar{r}_{i}, i=1, \ldots, m$, always has $r_{i}=1, i=1, \ldots, m$ (Nijmeijer and van der Schaft, 1990). Thus, if a discrete-time nonlinear system of the form of Eq. 1 has $r_{i}>1,\left(r_{i}-1\right) \Delta t$ represents the smallest plant deadtime between the manipulated inputs and the output $y_{i}$, whereas the additional delay $\Delta t$ is the time delay due to the sampling. Since, in a discrete-time setting, introduction of time delays simply increases the dimension and relative orders of the system, deadtime compensation in discrete time is much easier than in continuous time (especially for multivariable systems).

Definition 2. Consider a discrete-time, MIMO system of the form of Eq. 1 and assume that each output $y_{i}$ possesses a finite relative order $r_{i}$. The matrix

$$
\mathcal{C}(x, u) \triangleq\left[\begin{array}{c}
\frac{\partial}{\partial u} h_{1}^{r_{1}-1}[\Phi(x, u)] \\
\vdots \\
\frac{\partial}{\partial u} h_{m}^{r_{m}-1}[\Phi(x, u)]
\end{array}\right]
$$

is called the characteristic matrix of the system described by Eq. 1.

Throughout this article, it will be assumed that $\mathcal{C}(x, u)$ is nonsingular on $X \times U$. This assumption can always be guaranteed, as long as $\operatorname{det}[C(0,0)] \neq 0$, by appropriately defining the sets $X$ and $U$.

For a process of the form of Eq. 1 with finite relative orders and nonsingular characteristic matrix $\mathcal{C}[x(k), u(k)]$, Eq. 3 implies that the set of algebraic equations

$$
h_{i}^{r_{i}-1}[\Phi(x, u)]=y_{i}^{*}, \quad i=1, \ldots, m
$$

is locally solvable for the manipulated input vector $u$. The corresponding implicit function will be denoted by

$$
u=\Psi_{0}\left(x, y^{*}\right)
$$

where $y^{*}=\left[y_{1}^{*} \cdots y_{m}^{*}\right]^{T}$, and will be assumed to be well-defined and unique on $X \times h(X)$.

\section{Time-Delay Factorization of Nonlinear Systems}

The definition and interpretation of the relative orders $r_{1}$, $\ldots, r_{m}$ and the characteristic matrix $\mathfrak{e}(x(k), u(k))$ can be used to decompose a dynamic system of the form of Eq. 1 into two subsystems in series: (i) a "delay-free" subsystem, and (ii) a pure delay subsystem. This is established in the following theorem and shown in Figure 1 (in which $q$ represents the forward shift operator).

Proposition 1. If the system of Eq. 1 possesses finite relative orders and nonsingular characteristic matrix $\mathcal{C}(x, u)$ on $X \times h(X)$, then the system can be decomposed into the following two subsystems in series:

(a) the "delay-free" subsystem (whose inverse is physically realizable)

$$
\left\{\begin{aligned}
x(k+1) & =\Phi[x(k) u(k)] \\
y_{i}^{*}(k) & =h_{i}^{r_{i}-1}\{\Phi[x(k), u(k)]\}, \quad i=1, \ldots, m
\end{aligned}\right.
$$

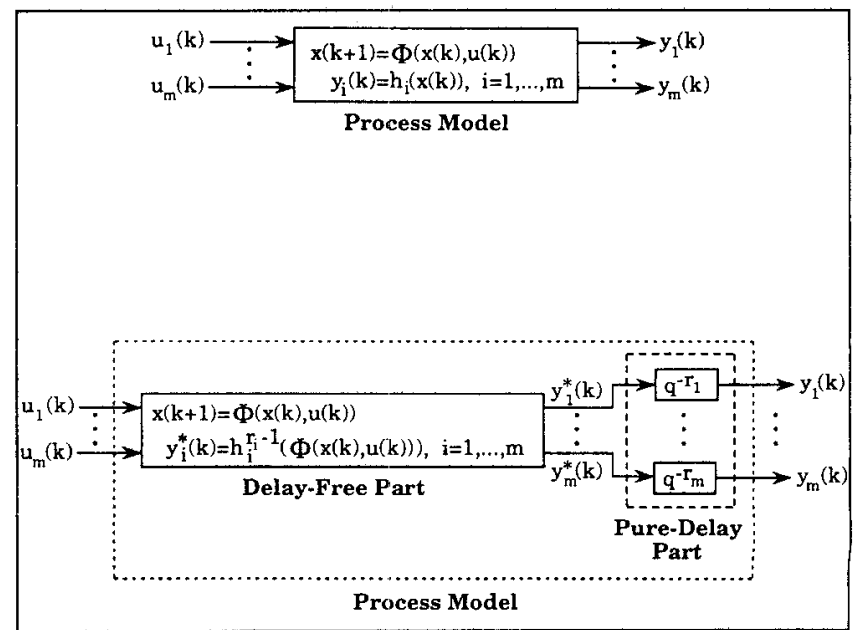

Figure 1. Decomposition of a nonlinear system into a delay-free part and a diagonal, pure-delay part. 
(b) the completely decoupled, pure-delay subsystem

$$
y_{i}(k)=y_{i}^{*}\left(k-r_{i}\right), \quad i=1, \ldots, m
$$

The proof was given by Soroush (1992).

This decomposition is a generalization of the standard factorization of linear discrete-time systems into an invertible (delay-free) part and a decoupled pure-delay part (Garcia and Morari, 1985a,b). Note that in Theorem 1, the term "delayfree" was used in the context that the inverse of the system under consideration is causal (physically realizable). Throughout the rest of this article the term "delay-free" will also be used in the same context.

As given in Proposition 1, because of the nonsingularity of the characteristic matrix, and therefore solvability of the algebraic equations in Eq. 4, an inverse to the delay-free subsystem (Eq. 6) can be constructed:

$$
\left\{\begin{aligned}
x(k+1) & =\Phi\left\{x(k), \Psi_{0}\left[x(k), y^{*}(k)\right]\right\} \\
u(k) & =\Psi_{0}\left[x(k), y^{*}(k)\right] .
\end{aligned}\right.
$$

In the case of a linear process with finite relative orders and with $n_{z}$ finite transmission zeros, the inverse system described by Eq. 8 will have $n_{z}$ poles at the process transmission zeros and $\left(n-n_{z}\right)$ poles at the origin. Consequently, in this case, the inverse system of Eq. 8 will be stable, if all the finite transmission zeros of the process are inside the unit circle. This motivates the definitions given in the next subsection, which are used to characterize minimum-phase behavior for nonlinear systems.

\section{Minimum-phase behavior}

For a linear system, the delay-free part of the system is said to be minimum phase, if all the finite transmission zeros of the system lie inside the unit circle. For a nonlinear system, notions of transmission zero and pole, however, cannot be used, and therefore minimum-phase behavior must be characterized in terms of the stability of the inverse dynamics.

Definition 3. Given a discrete-time nonlinear system of the form of Eq. 1, its delay-free part is said to be locally minimum phase, if the dynamics

$$
x(k+1)=\Phi\left\{x(k), \Psi_{0}\left[x(k), y^{*}(k)\right]\right\}
$$

is locally asymptotically stable. Otherwise, we will say that it is locally nonminimum phase.

The local asymptotic stability of the system described by Eq. 9 can be checked, for example, via Lyapunov's first method, by calculating the eigenvalues of the Jacobian of the system evaluated at the equilibrium point. Using the definition of $\Psi_{0}$ (Eqs. 4 and 5) and the implicit function theorem, one can easily see that the Jacobian of the system of Eq. 9 at $\left(x_{0}, y_{0}\right)=(0,0)$ is equal to

$$
\mathfrak{J}_{0}(x, u)=\left[\frac{\partial \Phi(x, u)}{\partial x}\right]
$$

$$
-\left[\frac{\partial \Phi(x, u)}{\partial u}\right][\mathcal{E}(x, u)]^{-1}\left[\begin{array}{c}
\frac{\partial}{\partial x} h_{1}^{r_{1}-1}[\Phi(x, u)] \\
\vdots \\
\frac{\partial}{\partial x} h_{m}^{r_{m}-1}[\Phi(x, u)]
\end{array}\right]
$$

evaluated at $(x, u)=(0,0)$. If all the eigenvalues of the Jacobian matrix $\mathcal{J}_{0}(x, u)$ are in the interior of the unit circle, the dynamics of Eq. 9 will be locally asymptotically stable in a neighborhood of the origin.

Definition 4. Given a discrete-time nonlinear system of the form of Eq. 1, its delay-free part is said to be locally hyperbolically minimum phase, if all the eigenvalues of the Jacobian matrix $\mathfrak{g}_{0}(x, u)$ evaluated at the equilibrium point, are in the interior of the unit circle.

Note that a locally hyperbolically minimum-phase delayfrec part is also locally minimum phase. However, the converse may not hold: the delay-free part of a system, whose corresponding Jacobian matrix $\mathfrak{J}_{0}(0,0)$ possesses some eigenvalues on the unit circle, can be locally minimum phase.

In the systems theory literature (see, e.g., Monaco and Normand-Cyrot, 1988), the notion of minimum phaseness is defined via the notion of zero dynamics, which tries to generalize the continuous-time notion of zero dynamics of Byrnes and Isidori (1985). Note, however, that the associated normal form of discrete-time systems is built on the manifold

$L^{*}=\left\{x \in X \mid h_{i}^{0}(x)=h_{i}^{1}(x)=\cdots=h_{i}^{r_{i}-1}(x)=0\right.$,

$$
i=1, \ldots, m\}
$$

rather than the entire state space $X$. Consequently, the normal form of Monaco and Normand-Cyrot is not connected with the (forced) inverse dynamics.

\section{Input-Output Linearizing State Feedback}

A static state-feedback law of the form

$$
u(k)=\Psi[x(k), v(k)]
$$

with $\operatorname{det}[\partial\{\Psi[x(k), v(k)]\} / \partial v] \neq 0$, which induces linear closed-loop input-output behavior, is sought. Here $v=\left[v_{1} \cdots\right.$ $\left.v_{m}\right]^{T}$ is the vector of reference inputs.

In particular, if linear closed-loop input-output dynamics of minimal order is desirable, this has the general form

$$
\left[\begin{array}{c}
y_{1}\left(k+r_{1}\right) \\
\vdots \\
y_{m}\left(k+r_{m}\right)
\end{array}\right]+\sum_{i=1}^{m} \sum_{\ell=1}^{r_{i}} \beta_{i \ell} y_{i}\left(k+r_{i}-\ell\right)=\mathcal{B}_{0}\left[\begin{array}{c}
v_{1}(k) \\
\vdots \\
v_{m}(k)
\end{array}\right],
$$

where $\beta_{i \ell}=\left[\beta_{i \ell}{ }^{1} \cdots \beta_{i \ell}^{m}\right]^{T}$ is an $m$-vector adjustable constant parameter and $\beta_{0}$ is a constant nonsingular $m \times m$ matrix. 
The input-output dynamics of Eq. 11, in the $z$-domain, is given by $y(z)=[Q(z)]^{-1} B_{0} v(z)$, where

$$
Q(z)=\operatorname{diag}\left\{z^{r_{i}}\right\}+\left[\left(\sum_{\ell=1}^{r_{1}} \beta_{1 \ell} z^{r_{1}-\ell}\right) \ldots\left(\sum_{\ell=1}^{r_{m}} \beta_{m \ell} z^{r_{m}-\ell}\right)\right]
$$

The matrix transfer function $G_{i}(z) \triangleq[Q(z)]^{-1} \mathcal{B}_{0}$ has $\left(r_{1}\right.$ $+\cdots+r_{m}$ ) poles at the roots of $\operatorname{det}[Q(z)]=0$ and no zeros: the determinantal degree of $G_{i}(z)$ is $\left(r_{1}+\cdots+r_{m}\right)$, which is the order of the closed-loop system.

The necessary static state feedback, which induces the input-output dynamics of Eq. 11, is easily calculated by using the definition and properties of the relative orders $r_{1}, \ldots, r_{m}$ and the characteristic matrix. It is the implicit function defined as the solution for $u(k)$ of the set of algebraic equations control is known for its poor robustness properties, and therefore it is of little practical importance.

The response of Eq. 15 is the fastest achievable closed-loop response in terms of tracking the reference inputs $v_{1}, \ldots, v_{m}$. On the other hand, the closed-loop response under the deadbeat controller (Eq. 14) is exactly the dynamics of pure-delay subsystem (Eq. 7), which is in agreement with Proposition 1. Thus, the closed-loop dynamics arising from the controller of Eq. 14

$$
x(k+1)=\Phi\left\{x(k), \Psi_{0}[x(k), v(k)]\right\}
$$

is also identical to the dynamics of the inverse of the delay. free part of the process (cf. Eq. 8). Therefore, a system of the form of Eq. 1 is locally minimum phase if and only if the closed-loop dynamics associated with the output deadbeat response is locally asymptotically stable (Monaco and Normand-Cyrot, 1988).

For practical purposes, we must use $B_{i \ell} \neq[0 \cdots 0]^{T}$ to obtain a sufficiently robust response. For this reason, one must analyze the closed-loop system arising from the general state-feedback law of Eq. 13:

$$
\left\{\begin{aligned}
x(k+1) & =\Phi\left[x(k), \Psi_{0}\left(x(k), \mathrm{B}_{0} v(k)-\sum_{i=1}^{m} \sum_{\ell=1}^{r_{i}} \beta_{i \ell} h_{i}^{r_{i}-\ell}[x(k)]\right)\right] . \\
y(k) & =h[x(k)]
\end{aligned}\right.
$$

$$
\begin{aligned}
{\left[\begin{array}{c}
h_{1}^{r_{1}-1}\{\Phi[x(k), u(k)]\} \\
\vdots \\
h_{m}^{r_{m}-1}\{\Phi[x(k), u(k)]\}
\end{array}\right]=\mathbb{B}_{0} v(k) } & \\
& -\sum_{i=1}^{m} \sum_{\ell=1}^{r_{i}} \beta_{i \ell} h_{i}^{r_{i}-\ell}[x(k)],
\end{aligned}
$$

which can be written as (using the definition of the function $\Psi_{0}$, given by Eqs. 4 and 5 ):

$$
u(k)=\Psi_{0}\left(x(k), \mathbb{\Theta}_{0} v(k)-\sum_{i=1}^{m} \sum_{\ell=1}^{r_{i}} \beta_{i \ell} h_{i}^{r_{i}-\ell}[x(k)]\right)
$$

Note that in the special case of $\beta_{i \ell}=[0 \cdots 0]^{T}, i=1, \ldots, m$, $\ell=1, \ldots, r_{i}$, and $\mathcal{B}_{0}=I_{m}$, the state feedback of Eq. 13 simplifies to

$$
u(k)=\Psi_{0}(x(k), v(k))
$$

and the resulting closed-loop input-output behavior to

$$
\left[\begin{array}{c}
y_{1}\left(k+r_{1}\right) \\
\vdots \\
y_{m}\left(k+r_{m}\right)
\end{array}\right]=\left[\begin{array}{c}
v_{1}(k) \\
\vdots \\
v_{m}(k)
\end{array}\right]
$$

which is an output deadbeat (time-optimal) response. Deadbeat control has been studied extensively in the systems theory literature (e.g., O'Reilly, 1981; Glad, 1987). Deadbeat

\section{Stability of the linearized $v-y$ system}

The input-output behavior of the closed-loop system (Eq. 17 ) is, of course, governed by the matrix transfer function $G_{i}(z)$, and therefore the input-output stability and performance characteristics of the closed-loop system (Eq. 17) are determined by the roots of the characteristic equation $\operatorname{det}[Q(z)]=0$. The local, asymptotic, internal stability of the closed-loop system (Eq. 17) depends on the eigenvalues of its Jacobian, evaluated at the equilibrium point. Using the definition of $\Psi_{0}$ (Eqs. 4 and 5) and the implicit function theorem, one can easily see that the Jacobian of Eq. 17 is given by

$$
\mathfrak{d}_{\beta}(x, u)=\left[\frac{\partial \Phi(x, u)}{\partial x}\right]-\left[\frac{\partial \Phi(x, u)}{\partial u}\right][\mathcal{C}(x, u)]^{-1}
$$

$$
\left\{\left[\begin{array}{c}
\frac{\partial}{\partial x} h_{1}^{r_{1}-1}[\Phi(x, u)] \\
\vdots \\
\frac{\partial}{\partial x} h_{m}^{r_{m}-1}[\Phi(x, u)]
\end{array}\right]+\sum_{i=1}^{m} \sum_{\ell=1}^{r_{i}} \beta_{i \ell} \frac{\partial h_{i}^{r_{i}-\ell}[x(k)]}{\partial x}\right\}
$$

Thus, if the eigenvalues of $\oiint_{\beta}(0,0)$ are in the interior of the unit circle, the closed-loop system of Eq. 17 is guaranteed to be locally internally stable. Precise conditions for the closedloop stability are given in the following proposition.

Proposition 2. Consider a system of the form of Eq. 1 with finite relative orders and nonsingular characteristic matrix, which is subject to a state feedback of the form of Eq. 13, where parameters $\beta_{i p}$ are chosen such that the roots of 
$\operatorname{det}[Q(z)]=0$ are in the interior of the unit circle. If the delay-free part of the system of Eq. 1 is locally hyperbolically minimum phase, the closed-loop system (Eq. 17) will be locally asymptotically stable. The proof is given in (Soroush, 1992).

As a consequence of this proposition, the input-output linearizing state feedback (Eq. 13) can form a basis for controller design for all locally hyperbolically minimum phase systems of the general form of Eq. 1 .

\section{Globally Linearizing Control Structure}

In the previous section, we saw that when a nonlinear system of the form of Eq. 1 is subjected to the state feedback of Eq. 13, the closed-loop system becomes linear in an input-output sense, meaning that the relation between $v$ and $y$ is described by a set of linear difference equations. The offsetless tracking of setpoints in the presence of model errors and step disturbances can be ensured by placing a linear multivariable controller with integral action around the linear $v-y$ system. This idea leads to the GLC structure (Kravaris and Soroush, 1990; Soroush and Kravaris, 1992b), which is depicted in Figure 2a. The linear controller of the GLC structure has been referred to as the external controller.

Requesting a desirable overall closed-loop behavior (setpoint-to-output relation)

$$
y(z)=G_{0}(z) y_{s p}(z)
$$

where $y_{s p}=\left[y_{s p_{1}} \cdots y_{s p_{m}}\right]^{T}$ is the vector of output setpoints, one can easily calculate the necessary external controller $G_{e}(z)$. In particular, if

$$
G_{\ell}(z)=[R(z)]^{-1} R(1),
$$

where $R(z)$ is a polynomial matrix in $z$ with $R(1)$ nonsingular and finite, then the necessary external controller $G_{e}(z)$ is given by

$$
G_{e}(z)=\mathbb{B}_{0}^{-1} Q(z)\left\{[R(1)]^{-1} R(z)-I_{m}\right\}^{-1}
$$

which possesses integral action: $G_{e}(z)$ has a pole at $z=1$. Causality of the external controller $G_{e}(z)$ is guaranteed as long as the matrix $R(z)$ is chosen to be a column reduced polynomial matrix with the column degrees $r_{1}, \ldots, r_{m}$ :

$R(z)=\operatorname{diag}\left\{z^{r_{i}}\right\}+\left[\left(\sum_{\ell=1}^{r_{1}} \gamma_{1 \ell} z^{r_{1}-\ell}\right) \ldots\left(\sum_{\ell=1}^{r_{m}} \gamma_{m \ell} z^{r_{m}-\ell}\right)\right]$,

where $\gamma_{i \ell}=\left[\gamma_{i \ell}^{1} \cdots \gamma_{i \ell}^{m}\right]^{T}$ are $m$-vectors of constant parameters with $\operatorname{det}[R(1)] \neq 0$. In Eq. 22 , the leading coefficient matrix was set to be the identity matrix without loss of generality, in view of the scaling of $G_{o}(z)$, given in Eq. 20 .

To implement the external controller $G_{e}(z)$, we should calculate a state-space realization of the matrix transfer function $G_{e}(z)$. Minimal-order state-space realizations can be derived using standard methods from linear systems theory (e.g.,

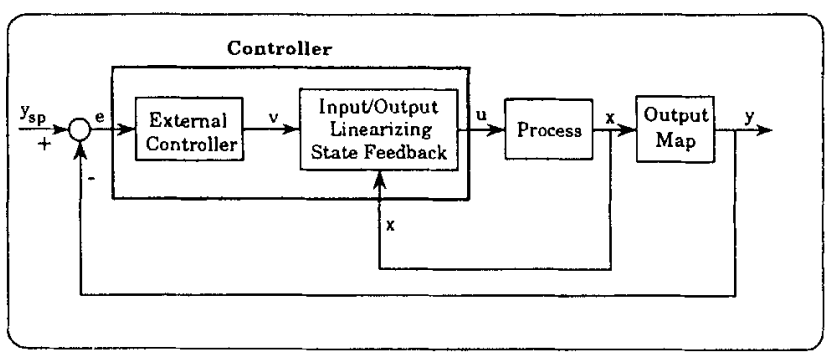

Figure 2a. Basic GLC structure.

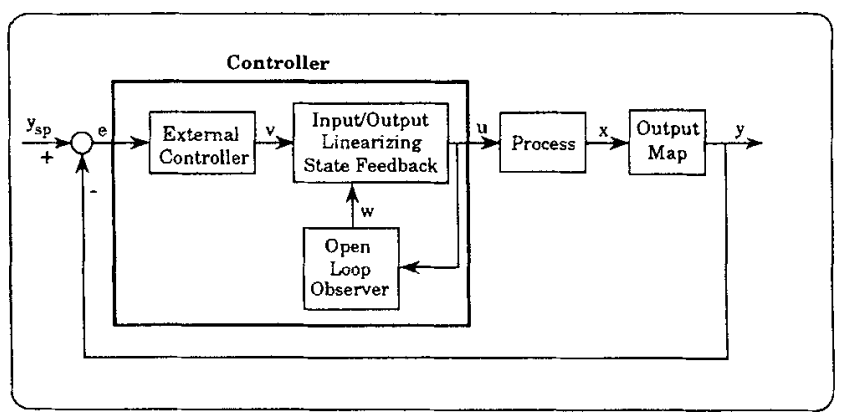

Figure 2b. Error-feedback GLC structure.

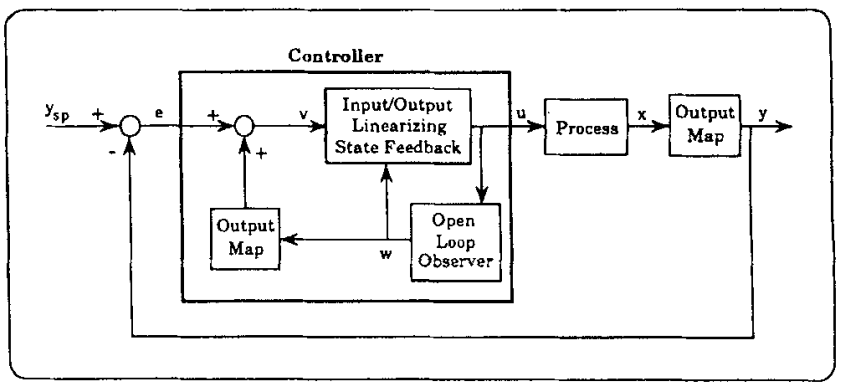

Figure 2c. Error-feedback (reduced-order) GLC structure.

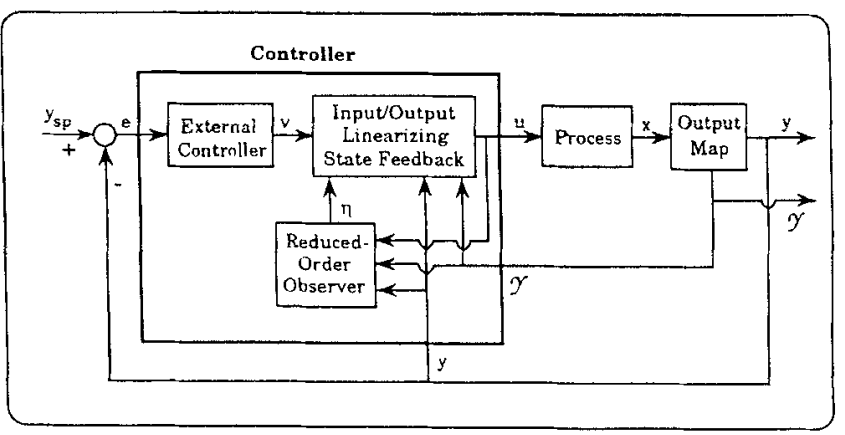

Figure 2d. Mixed error- and output-feedback GLC structure.

Chen, 1984). For completeness, a minimal-order, state-space realization of the external controller (defined by Eqs. 21 and 22) is given in the Appendix; it has the compact form:

$$
\begin{aligned}
\xi(k+1) & =A_{e} \xi(k)+B_{e} e(k) \\
\mathbb{B}_{\emptyset} \nu(k) & =C_{e} \xi(k)+D_{e} e(k),
\end{aligned}
$$


where $\xi(k)=\left[\xi_{1}^{(1)}(k) \cdots \xi_{r_{1}}^{(1)}(k) \cdots: \xi_{1}^{(m)}(k) \cdots \xi_{r_{m}}^{(m)}(k)\right]^{T}, e(k)$ $=\left[e_{1}(k) \cdots e_{m}(k)\right]^{T}=y_{s p}(k)-y(k)$, and $A_{e}, B_{e}, C_{e}$, and $D_{e}$ are constant matrices, which are defined in Appendix $\mathrm{A}$. Equation 23 is a minimal-order state-space realization, because both the order of $G_{e}(z)$ [degree-in-z of the determinant of $R(z)$ ] and the order of the state-space realization are $\left(r_{1}+\cdots+r_{m}\right)$.

\section{Synthesis of mixed error-and state-feedback controllers}

In the case that all state variables are measured on-line, the state feedback of Eq. 13 and the external controller (Eq. 23) are implemented to induce the desired linear overall closed-loop response of Eqs. 20 and 22. This motivates the following theorem.

Theorem 1. Consider the nonlinear process described by Eq. 1 with finite relative orders and nonsingular characteristic matrix $\mathcal{C}(x, u)$ on $X \times U$. Then, the dynamic system

\section{Synthesis of error-feedback controllers}

In many practical situations, complete on-line state information is not available. Quite often, only the outputs are measured on-line and this motivates the synthesis of nonlinear output-feedback control laws, which can induce a closedloop input-output behavior of the form of Eq. 25 .

In the case of a process operating only within the domain of attraction of an open-loop stable equilibrium point, state variables can be reconstructed by using an open-loop observer: $w(k+1)=\Phi[w(k), u(k)]$, where $w$ is the vector of state estimates. The state estimates $w_{1}, \ldots, w_{n}$ can then be used in the input-output linearizing state feedback of Eq. 13 in conjunction with the external controller of Eq. 23, leading to an error-feedback controller, which induces the closed-loop dynamics of Eq. 25. The synthesis formula of the resulting error-feedback controller is given in the following theorem.

Theorem 2. Consider the nonlinear process described by Eq. 1 with finite relative orders and nonsingular characteristic matrix $\mathcal{C}(x, u)$ on $X \times U$. Then, the dynamic system

$\left\{\begin{aligned} \xi(k+1) & =A_{e} \xi(k)+B_{e} e(k) \\ u(k) & =\Psi_{0}\left(x(k), C_{e} \xi(k)+D_{e} e(k)-\sum_{i=1}^{m} \sum_{\ell=1}^{r_{i}} \beta_{i \ell} h_{i}^{r_{i}-\ell}[x(k)]\right),\end{aligned}\right.$

$$
\left\{\begin{aligned}
\xi(k+1) & =A_{e} \xi(k)+B_{e} e(k) \\
w(k+1) & =\Phi\left[w(k), \Psi_{0}\left(w(k), C_{e} \xi(k)+D_{e} e(k)-\sum_{i=1}^{m} \sum_{\ell=1}^{r_{i}} \beta_{i \ell} h_{i}^{r_{i}-\ell}[w(k)]\right)\right] \\
u(k) & =\Psi_{0}\left(w(k), C_{e} \xi(k)+D_{e} e(k)-\sum_{i=1}^{m} \sum_{\ell=1}^{r_{i}} \beta_{i \ell} h_{i}^{r_{i}-\ell}[w(k)]\right)
\end{aligned}\right.
$$

where $\Psi_{0}(.,$.$) is defined by Eqs. 4$ and 5 , and $A_{e}, B_{e}, C_{e}$, and $D_{e}$ are the system matrices of the state-space realization of Eq. 23, represents an $\left(r_{1}+\cdots+r_{m}\right)$ th order state-space realization of a dynamic mixed error- and state-feedback controller that induces the closed-loop input-output behavior described by Eqs. 20 and 22:

$$
\left[\begin{array}{c}
y_{1}\left(k+r_{1}\right) \\
\vdots \\
y_{m}\left(k+r_{m}\right)
\end{array}\right]+\sum_{i=1}^{m} \sum_{\ell=1}^{r_{i}} \gamma_{i \ell} y_{i}\left(k+r_{i}-\ell\right)=R(1)\left[\begin{array}{c}
y_{s p_{1}}(k) \\
\vdots \\
y_{s p_{m}}(k)
\end{array}\right]
$$

The proof is given by Soroush (1992). The block diagram of the mixed error- and state-feedback controller (Eq. 24) is depicted in Figure 2a, which is the "Basic" GLC structure. where $\Psi_{0}(.,$.$) is defined by Eqs. 4$ and 5 , and $A_{e}, B_{e}, C_{e}$ and $D_{e}$ are the system matrices of the state-space realization of Eq. 23, represents an $\left(n+r_{1}+\cdots+r_{m}\right)$ th order state-space realization of a dynamic error-feedback controller that induces the closed-loop input-output behavior of Eq. 25 , if the process and its model are initialized consistently $[x(0)=w(0)]$. The proof is given by Soroush (1992).

In the controller of Theorem 2, if the external controller and the process model (observer) are initialized such that

$$
\xi_{\ell}^{(i)}(0)=h_{i}^{r_{i}-\ell}[w(0)], \ell=1, \ldots, r_{i}, i=1, \ldots, m
$$

the controller realization of Eq. 26 will have $r_{1}+\cdots+r_{m}$ redundant modes; the modes of the external controller will be the same as $r_{1}+\cdots+r_{m}$ modes of the observer subsystem. In this case, the elimination of the redundant dynamics of the external controller leads to a reduced-order realization of the controller of Theorem 2 . The resulting controller is given in the following corollary.

Corollary 1 (Reduced-Order Realization). Under the assumptions of Theorem 2, the dynamic system

$$
\left\{\begin{aligned}
w(k+1) & =\Phi\left[w(k), \Psi_{0}\left(w(k), R(1)[e(k)+h[w(k)]]-\sum_{i=1}^{m} \sum_{\ell=1}^{r_{i}} \gamma_{i \ell} h_{i}^{r_{i}-\ell}[w(k)]\right)\right] \\
u(k) & =\Psi_{0}\left(w(k), R(1)[e(k)+h[w(k)]]-\sum_{i=1}^{m} \sum_{\ell=1}^{r_{i}} \gamma_{i \ell} h_{i}^{r_{i}-\ell}[w(k)]\right),
\end{aligned}\right.
$$


where $\Psi_{0}(.,$.$) is defined by Eqs. 4$ and 5 , represents an $n$th order realization of a dynamic error-feedback controller, which induces the closed-loop input-output behavior of Eq. 25 , if the process model and the external controller are initialized such that $w(0)=x(0)$ and $\xi_{\ell}^{(i)}(0)=h_{i}^{r_{i}-\ell}(w(0)), \ell=1$, $\ldots, r_{i}, i=1, \ldots, m$. The proof is given in Soroush (1992).

The controller realizations of Eqs. 26 and 27 represent a dynamic error-feedback controller (the only input to the controller is the crror) with integral action (Eq. 27 is a reducedorder realization of Eq. 26). The error-feedback GLC structures are depicted in Figures $2 \mathrm{~b}$ and $2 \mathrm{c}$.

\section{Synthesis of mixed error- and output-feedback controllers}

The control laws of Eqs. 26 and 27 are applicable to the processes operating only within the domain of attraction of an open-loop stable equilibrium point. Otherwise, any error in the observer initialization will grow indefinitely, leading to internal instability. This motivates the development of alternative state-reconstruction techniques.

In the present subsection, we will explore the use of the reduced-order observer, which simulates a stable subsystem of the process model, driven by the output measurements and process inputs. When such an observer is coupled with the input-output linearizing state feedback and the external controller, it gives rise to a mixed-error and output-feedback control law. To make the development sufficiently general, we will include the possibility of available secondary measurements, in addition to the measurements of the controlled output (primary measurements).

Definition 5. For a process of the form of Eq. 1, the measurable process variables $\mathcal{Y}_{1}, \ldots, \mathcal{Y}_{s}$ will be called the secondary outputs, if they are algebraic functions of the state variables, $\mathcal{Y}_{i}=\mathcal{H}_{i}(x), i=1, \ldots, s$, such that the $(m+s) \times n$ matrix

$$
\left[\begin{array}{c}
\frac{\partial \mathfrak{H C}(x)}{\partial x} \\
\frac{\partial h(x)}{\partial x}
\end{array}\right]
$$

where $\mathscr{H}(x)=\left[\begin{array}{llll}\mathcal{H}_{1}(x) & \cdots & \mathfrak{H}_{s}(x)\end{array}\right]^{T}$ has $(s+m)$ linearly independent rows, $\forall x \in X$.

The nonsingularity of the characteristic matrix on $X \times U$ is a sufficient condition for output controllability, and therefore a sufficient condition for the matrix $[\partial h(x) / \partial x]$ to have $m$ linearly independent rows, $\forall x \in X$. If a process has a nonsingular characteristic matrix and the condition of Eq. 28 is not satisfied, then there is at least one $\mathcal{Y}_{i}$ that is a "redundant" process measurement; this can be resolved by dropping at least one of the extra measurements as secondary output(s).

Consider a process of the form of Eq. 1 with $s(s \geq 0)$ secondary outputs:

$$
\left\{\begin{array}{rlrl}
x(k+1) & =\Phi[x(k), u(k)] & & \\
y_{i}(k) & =h_{i}[x(k)], & & i=1, \cdots, m \\
\mathcal{Y}_{i}(k) & =\mathfrak{H C}_{i}[x(k)], & i=1, \cdots, s .
\end{array}\right.
$$

To such a process, we apply the coordinate transformation:

$$
\left[\begin{array}{c}
\eta_{1} \\
\vdots \\
\eta_{n-s-m} \\
\mathcal{Y} \\
y
\end{array}\right]=\mathfrak{J}(x)=\left[\begin{array}{c}
x_{1} \\
\vdots \\
x_{n-s-m} \\
\mathfrak{H C}(x) \\
h(x)
\end{array}\right]
$$

where $x_{1}, \ldots, x_{n-s-m}$ are $(n-s-m)$ state variables of the system of Eq. 29, which satisfy the condition:

$$
\operatorname{det}\left(\frac{\partial}{\partial x}\left[\begin{array}{c}
x_{1} \\
\vdots \\
x_{n-s-m} \\
\mathcal{H}(x) \\
h(x)
\end{array}\right]\right) \neq 0, \quad \forall x \in X
$$

Note that the condition of Eq. 28 guarantees the existence of the $(n-s-m)$ state variables $x_{1} \cdots x_{n-s-m}$, which satisfy the condition of Eq. 31. Because of the condition of Eq. 31, the coordinate transformation of Eq. 30 is invertible on $X$.

The system of Eq. 29, in the new coordinates of Eq. 30, takes the form:

$$
\left\{\begin{aligned}
\eta_{1}(k+1) & =F_{1}[\eta(k), \mathcal{Y}(k), y(k), u(k)] \\
& \vdots \\
\eta_{n-s-m}(k+1) & =F_{n-s-m}[\eta(k), \mathcal{Y}(k), y(k), u(k)] \\
\mathcal{Y}(k+1) & =\Omega[\eta(k), \mathcal{Y}(k), y(k), u(k)] \\
y(k+1) & =\phi[\eta(k), \mathcal{Y}(k), y(k), u(k)] \\
y(k) & =H[y(k)]
\end{aligned}\right.
$$

where $\eta=\left[\eta_{1} \cdots \eta_{n-s-m}\right]^{T}$,

$$
\begin{gathered}
F_{\ell}(\eta, \mathcal{Y}, y, u)=\Phi_{\ell}\left\{\mathcal{J}^{-1}\left([\eta \mathcal{Y} y]^{T}\right), u\right\}, \\
\ell=1, \ldots, n-s-m, \\
\Omega(\eta, \mathcal{Y}, y, u)=\mathcal{H}\left(\Phi\left\{\mathcal{J}^{-1}\left([\eta \mathcal{Y} y]^{T}\right), u\right\}\right), \\
\phi(\eta, \mathcal{Y}, y, u)=h\left(\Phi\left\{\mathfrak{J}^{-1}\left([\eta \mathcal{Y} y]^{T}\right), u\right\}\right) .
\end{gathered}
$$

In the system of Eq. 32, the last $(m+s)$ state variables are measured on-line, whereas the first $(n-m-s)$ state variables are not and can be reconstructed by simulating the first $(n-m-s)$ state equations, which are driven by the primary and secondary outputs and the manipulated inputs. Therefore, the reduced-order observer:

$$
\begin{aligned}
\eta_{1}(k+1) & =F_{1}[\eta(k), \mathcal{Y}(k), y(k), u(k)] \\
& \vdots \\
\eta_{n-m-s}(k+1) & =F_{n-m-s}[\eta(k), \mathcal{Y}(k), y(k), u(k)]
\end{aligned}
$$

or, in a compact form, $\eta(k+1)=F[\eta(k), \mathcal{Y}(k), y(k), u(k)]$, is used to reconstruct the state variables $x_{1}, \cdots, x_{n-s-m}$. The state estimates $\eta_{1}, \ldots, \eta_{n-m-s}$ can now be used in an input-output linearizing state feedback, synthesized for the system of Eq. 32. This idea is precisely formulated in the following theorem. 
Theorem 3. Consider the system described by Eq. 32 with $s$ secondary outputs $\mathcal{Y}_{1}, \ldots, \mathcal{Y}_{s}(s \geq 0)$, finite relative orders, and nonsingular characteristic matrix $\mathfrak{e}(x, u)$ on $X \times U$. Then, the dynamic system

\section{Controller simplifications}

In this subsection, the feedback controllers of Theorems 2, 3 and 4 , and Corollary 1 are simplified by requesting desirable closed-loop responses, leading to the reduction in the

$$
\left\{\begin{aligned}
\xi(k+1) & =A_{e} \xi(k)+B_{e} e(k) \\
\eta(k+1) & =F\left\{\eta(k), \mathcal{Y}(k), y(k), \Psi_{0}\left(\left[\begin{array}{c}
\eta(k) \\
\mathcal{Y}(k) \\
y(k)
\end{array}\right], C_{e} \xi(k)+D_{e} e(k)-\sum_{i=1}^{m} \sum_{\ell=1}^{r_{i}} \beta_{i \ell} H_{i}^{r_{i}-\ell}\left(\left[\begin{array}{c}
\eta(k) \\
\mathcal{Y}(k) \\
y(k)
\end{array}\right]\right)\right)\right\} \\
u(k) & =\Psi_{0}\left(\left[\begin{array}{c}
\eta(k) \\
\mathcal{Y}(k) \\
y(k)
\end{array}\right], C_{e} \xi(k)+D_{e} e(k)-\sum_{i=1}^{m} \sum_{\ell=1}^{r_{i}} \beta_{i \ell} H_{i}^{r_{i}-\ell}\left(\left[\begin{array}{c}
\eta(k) \\
\mathcal{Y}(k) \\
y(k)
\end{array}\right]\right)\right),
\end{aligned}\right.
$$

where $\Psi_{0}(.,$.$) is defined by Eqs. 4$ and 5 , and $A_{e}, B_{e}, C_{e}$, and $D_{e}$ are the system matrices of the state-space realization of Eq. 23, represents an $\left(n+r_{1}+\cdots+r_{m}-m-s\right)$ th order state-space realization of a dynamic mixed error- and output-feedback controller that induces the input-output behavior of Eq. 25 to the closed-loop system, if the reducedorder observer and process are initialized consistently $[\eta(0)$ $\left.=\left[x_{1}(0) \cdots x_{n-s-m}(0)\right]^{T}\right]$. The proof is given by Soroush (1992).

The controller realizations of Eq. 34 represents a dynamic mixed error- and output-feedback controller with integral action. The overall control structure as well as the various parts of the controller are shown in Figure 2d. This controller structure is the mixed error-and output-feedback GLC structure.

\section{Closed-loop stability}

Consider the conditions: (i) the $m$-vector parameters $\gamma_{i \ell}$ are chosen such that the roots of the characteristic equation $\operatorname{det}[R(z)]=0$ lie inside the unit circle; (ii) the $m$-vector parameters $\beta_{i \ell}$ are chosen such that the roots of the characteristic equation $\operatorname{det}[Q(z)]=0$ lie inside the unit circle; (iii) the process is locally hyperbolically minimum phase; (iv) the process is locally asymptotically open-loop stable; and (v) the system of Eq. 33 is locally asymptotically open-loop stable.

The overall closed-loop system (under the controllers of Theorems 1, 2, and 3, and Corollary 1) will be input-output stable, if the condition (i) holds.

For an input-output stable closed-loop system, the local internal stability will be ensured (a) under the controller of Theorem 1, if (ii) and (iii) hold; (b) under the controller of Theorem 2, if (ii), (iii) and (iv) hold; (c) under the controller of Theorem 3, if (ii), (iii) and (v) hold; (d) under the controller of Corollary 1, if (iii) and (iv) hold. number of adjustable parameters while leaving enough flexibility to "shape" the closed-loop response.

(I) First-Order-Plus-Deadtime Response. This type of response is obtained by placing $\left(r_{1}+\cdots+r_{m}-m\right)$ poles at the origin and leaving the remaining $m$ poles adjustable, which corresponds to setting:

$$
\gamma_{i 1} \neq[0 \cdots 0]^{T}, \quad \gamma_{i 2}=\cdots=\gamma_{i r_{i}}=[0 \cdots 0]^{T}, \quad i=1, \ldots, m .
$$

The requested desirable closed-loop input-output behavior has the form:

$$
\begin{array}{r}
{\left[\begin{array}{c}
y_{1}\left(k+r_{1}\right) \\
\vdots \\
y_{m}\left(k+r_{m}\right)
\end{array}\right]+\left[\gamma_{11} \cdots \gamma_{m 1}\right]} \\
{\left[\begin{array}{c}
y_{1}\left(k+r_{1}-1\right) \\
\vdots \\
y_{m}\left(k+r_{m}-1\right)
\end{array}\right]} \\
=\left\{I_{m}+\left[\gamma_{11} \cdots \gamma_{m 1}\right]\right\} y_{s p}(k)
\end{array}
$$

or, in the $z$-domain, $y(z)=\operatorname{diag}\left\{z^{-r_{i}}\right\}\left(I_{m}-\alpha z^{-1}\right)^{-1}\left(I_{m}-\right.$ a) $y_{s p}(z)$, where $\alpha \triangleq-\left[\gamma_{11} \cdots \gamma_{m 1}\right]$. The matrix $\left(I_{m}-\alpha_{z}^{-1}\right)^{-1}$ $\left(I_{m}-\alpha\right)$ is the transfer function of a multivariable first-order filter, and therefore the controller that leads to such a response can find a nice internal model control (IMC) interpretation in linear systems (Garcia and Morari, 1985a,b). Finally, practical experience from linear systems has shown that although this kind of response has only $m \times m$ tunable parameters, it gives enough flexibility to the designer. The relative magnitudes of off-diagonal elements with respect to the diagonal elements of the matrix $\alpha$ are measures of interactions between the setpoints and outputs. When the $\gamma_{i \ell} s$ are chosen according to Eq. 35, the controller of Eq. 27 simplifies into

$$
\left\{\begin{array}{c}
w(k+1)=\Phi\left(w(k), \Psi_{0}\left(w(k),\left(I_{m}-\alpha\right)[e(k)+h(w(k))]+\alpha\left[\begin{array}{c}
h_{1}^{r_{1}-1}(w(k)) \\
\vdots \\
h_{m}^{r_{m}-1}(w(k))
\end{array}\right]\right)\right) \\
u(k)=\Psi_{0}\left(w(k),\left(I_{m}-\alpha\right)[e(k)+h(w(k))]+\alpha\left[\begin{array}{c}
h_{1}^{r_{1}-1}(w(k)) \\
\vdots \\
h_{m}^{r_{m}-1}(w(k))
\end{array}\right]\right)
\end{array}\right.
$$


(II) Complete Input-Output Decoupling. One common design specification is to request a completely decoupled closed-loop response:

$$
\begin{array}{r}
y_{i}\left(k+r_{i}\right)+\sum_{\ell=1}^{r_{i}} \gamma_{i \ell}^{i} y_{i}\left(k+r_{i}-\ell\right)=\left(1+\sum_{\ell=1}^{r_{i}} \gamma_{i \ell}^{i}\right) y_{s p_{i}}(k), \\
i=1, \ldots, m
\end{array}
$$

or, in the $z$-domain, $y_{i}(z) / y_{s p_{i}}(z)=\left(1+\gamma_{i 1}^{i}+\cdots+\gamma_{i r}^{i}\right) /\left(z^{r_{i}}+\right.$ $\left.\gamma_{i 1}^{i} z^{r_{i}-1}+\cdots+\gamma_{i r_{i}}^{i}\right), i=1, \ldots, m$. The controllers of Eqs. 24, 26, and 34 induce the input-output response of Eq. 38, when the tunable parameters $\gamma_{i j}$ are chosen according to $\gamma_{i j}^{\ell}=0$, $\ell \neq i, \ell=1, \ldots, m, j=1, \ldots, r_{i}, i=1, \ldots, m$. Alternatively, it is possible to request triangular (partial) decoupling in closed-loop on the basis of a hierarchization of the importance of the controlled outputs.

(III) First-Order-Plus-Deadtime Response and Complete Input-Output Decoupling. This closed-loop response has the characteristics of both responses of Eqs. 36 and 38 ( $m$ decoupled first-order-plus-dead-time responses): $y_{i}\left(k+r_{i}\right)-\alpha_{i} y_{i}(k$ $\left.+r_{i}-1\right)=\left(1-\alpha_{i}\right) y_{s p}(k), i=1, \ldots, m$ or, in the $z$-domain, $y_{i}(z) / y_{s p_{i}}(z)=z^{-r_{i}}\left(1-\alpha_{i}\right) /\left(1-z^{-1} \alpha_{i}\right), i=1, \ldots, m$, which is obtained by setting $\gamma_{i 2}=\cdots=\gamma_{i r}=[0 \cdots 0]^{T}, i=1, \ldots, m$, and $\alpha=-\left[\gamma_{11} \cdots \gamma_{m 1}\right]=\operatorname{diag}\left\{\alpha_{i}\right\}$ with $\operatorname{det}(\alpha) \neq 0$.

\section{Implementation issues}

The input-output linearizing state-feedback of Eq. 13, which served as a basis in the nonlinear controller design approach of this article, may correspond to an implicit function. In such a case, the controller action should be calculated numerically by solving the set of algebraic equations:

$$
\left[\begin{array}{c}
h_{1}^{r_{1}-1}\{\Phi[x(k), u(k)]\} \\
\vdots \\
h_{m}^{r_{m}-1}\{\Phi[x(k), u(k)]\}
\end{array}\right]=\mathcal{B}_{0} v(k)
$$

$$
-\sum_{i=1}^{m} \sum_{\ell=1}^{r_{i}} \beta_{i \ell} h_{i}^{r_{i}-\ell}(x(k))
$$

for $u(k)$. Note that because of nonsingularity of the characteristic matrix, the solution for $u(k)$ will be at least locally unique. The numerical calculation of the controller action will not be computationally expensive, compared to the computational effort needed in MPC for solving an optimization problem on-line.

Except for the controller of Corollary 1, the feedback controllers derived in this article possess two sets of tunable parameters, $\beta_{i \ell}$ and $\gamma_{i \ell}$. While the performance characteristics and stability of the overall closed-loop system (input-output dynamics describing the relation between the setpoints and controlled outputs) are dependent on the adjustable parameters $\gamma_{i \ell}$ and are independent of the adjustable parameters $\beta_{i \ell}$, the dynamics of the hidden modes of the closed-loop system depends on the parameters $\beta_{i \ell}$. In addition to the requirement that the roots of $\operatorname{det}[Q(z)]=0$ should lie inside the unit circle, the parameters $\beta_{i \ell}$ should be chosen such that the robustness of the closed-loop system is ensured. In particular, enough care must be taken to ensure that the hidden modes are slower than the unmodeled dynamics. Experimental and simulation studies have shown that the parameters $\beta_{i \ell}$ have a strong effect on the robustness of the closedloop system.

\section{Connections to model predictive control}

While the connections between the derived controllers of Theorems 1, 2 and 3, and MPC are not transparent, it is straightforward to show that the controller of Eq. 37 (controller of Corollary 1 when the tunable parameters $\gamma_{i \ell}$ are chosen according to Eq. 35 ) is exactly a nonlinear multivariable model algorithmic controller (MAC) for unconstrained nonlinear processes. To show this equivalence, one needs to follow an approach similar to the one used in (Soroush and Kravaris, 1992b), wherein for unconstrained SISO processes, a nonlinear version of the linear MAC (Richalet et al., 1978; Mehra and Rouhani, 1980) was derived. The details of the MIMO MAC derivation can be found in Soroush (1992). The equivalence of the controller of Eq. 37 and the nonlinear MAC implies that

- under the nonlinear MAC, the closed-loop system is partially governed by the zero dynamics of the process, which is the nonlinear analog of placing a subset of closed-loop poles at the finite zeros of a process by a linear MAC.

- an unconstrained nonlinear model predictive controller (with (a) prediction horizons of one sampling period beyond the minimum deadtimes, (b) control horizons of one, and (c) a quadratic performance index without any penalties on controller action), is exactly an input-output linearizing feedback controller.

These theoretical results will be described in detail in a forthcoming publication. The controller of Eq. 37 can also be interpreted/implemented as a Smith predictor along the lines of our SISO results (Soroush and Kravaris, 1992b). For brevity, the details are not given here and can be found elsewhere (Soroush, 1992).

\section{Illustrative Example: Application to a CSTR}

To illustrate the application and performance of the derived control laws, we consider a CSTR example similar to the one used by Daoutidis et al. (1991), in which the following parallel reactions

$$
\begin{gathered}
A \stackrel{k_{1}}{\rightarrow} U_{1} \\
A \stackrel{k_{-1}}{\leftarrow} U_{1} \\
A \stackrel{k_{2}}{\rightarrow} U_{2} \\
A \stackrel{k_{3}}{\rightarrow} U_{3} \\
A \stackrel{k_{4}}{\rightarrow} \dot{U}_{4} \\
A \stackrel{k_{d}}{\rightarrow} D
\end{gathered}
$$

take place. $U_{1}, U_{2}, U_{3}$, and $U_{4}$ are undesirable side products, and $D$ is the desired product. The dependence of $k_{1}, k_{-1}$, $k_{2}, k_{3}, k_{4}$, and $k_{d}$ on temperature are given by $k_{i}=$ $Z_{i} \exp \left(-E_{a_{i}} / R T\right), \quad i=1,-1,2,3,4$ and $k_{d}=$ 
$Z_{d} \exp \left(-E_{a_{d}} / R T\right)$, respectively. It is assumed that the feed to the reactor does not contain $U_{1}, U_{2}, U_{3}, U_{4}$, or $D$.

\section{Mathematical model and control problem}

Mole balances on $A$ and $U_{1}$ and energy balance for the reactor (under standard assumptions) give the reactor model, which is of the form

$$
\left\{\begin{aligned}
\frac{d}{d t}\left[\begin{array}{c}
C_{A} \\
C_{U_{1}} \\
T
\end{array}\right] & =\left[\begin{array}{l}
f_{1}\left(C_{A}, C_{U_{1}}, T\right) \\
f_{2}\left(C_{A}, C_{U_{1}}, T\right) \\
f_{3}\left(C_{A}, C_{U_{1}}, T\right)
\end{array}\right]+\left[\begin{array}{c}
1 \\
\tau \\
0 \\
0
\end{array}\right] u_{1}+\left[\begin{array}{c}
0 \\
0 \\
\frac{1}{\rho c V}
\end{array}\right] u_{2} \\
y & =\left[\begin{array}{c}
C_{A} \\
T
\end{array}\right],
\end{aligned}\right.
$$

where $u_{1}=C_{A_{i}}, u_{2}=Q$, and

$f_{1}\left(C_{A}, C_{U_{1}}, T\right)=-\sum_{\ell=1}^{4} k_{\ell} C_{A}^{n_{\ell}}-k_{d} C_{A}^{n_{d}}+k_{-1} C_{U_{1}^{-1}}^{n_{-1}}-\frac{C_{A}}{\tau}$

$f_{2}\left(C_{A}, C_{U_{1}}, T\right)=k_{1} C_{A}^{n_{1}}-k_{-1} C_{A}^{n_{-1}}-\frac{C_{U_{1}}}{\tau}$

$f_{3}\left(C_{A}, C_{U_{l}}, T\right)=\frac{1}{\rho c_{p}}$

$\left[\sum_{\ell=1}^{4}\left(-\Delta H_{\ell}\right) k_{\ell} C_{A}^{n_{\ell}}+\left(-\Delta H_{d}\right) k_{d} C_{A}^{n_{d}}-\left(-\Delta H_{1}\right) k_{-1} C_{U_{1}^{-1}}^{n_{-1}}\right]$ $+\frac{T_{i}-T}{\tau}$

\begin{tabular}{|c|c|}
\hline $\begin{aligned} R & =8.345 \times 10^{0} \\
Z_{1} & =3.906 \times 10^{0} \\
Z_{-1} & =9.000 \times 10^{3} \\
Z_{2} & =3.906 \times 10^{0} \\
Z_{3} & =4.933 \times 10^{9} \\
Z_{4} & =4.933 \times 10^{9} \\
Z_{d} & =2.499 \times 10^{6}\end{aligned}$ & $\begin{array}{l}\mathrm{kJ} \cdot \mathrm{kmol}^{-1} \cdot \mathrm{K}^{-1} \\
\mathrm{~s}^{-1} \\
\mathrm{~s}^{-1} \\
\mathrm{~m}^{6} \cdot \mathrm{kmol}^{-2} \cdot \mathrm{s}^{-1} \\
\mathrm{~m}^{6} \cdot \mathrm{kmol}^{-2} \cdot \mathrm{s}^{-1} \\
\mathrm{~s}^{-1} \\
\mathrm{~m}^{3} \cdot \mathrm{kmol}^{-1}\end{array}$ \\
\hline $\begin{aligned} E_{a_{1}}=E_{a_{2}} & =2.000 \times 10^{4} \\
E_{a_{d}}=E_{a-1} & =6.000 \times 10^{4} \\
E_{a_{3}}=E_{a_{4}} & =1.000 \times 10^{5}\end{aligned}$ & $\begin{array}{l}\mathrm{kJ} \cdot \mathrm{kmol}^{-1} \\
\mathrm{~kJ} \cdot \mathrm{kmol}^{-1} \\
\mathrm{~kJ} \cdot \mathrm{kmol}^{-1}\end{array}$ \\
\hline $\begin{array}{l}-\Delta H_{1}=4.000 \times 10^{4} \\
-\Delta H_{2}=5.500 \times 10^{4} \\
-\Delta H_{3}=5.000 \times 10^{4} \\
-\Delta H_{4}=6.200 \times 10^{4} \\
-\Delta H_{d}=6.000 \times 10^{4}\end{array}$ & $\begin{array}{l}\mathrm{kJ} \cdot \mathrm{kmol}^{-1} \\
\mathrm{~kJ} \cdot \mathrm{kmol}^{-1} \\
\mathrm{~kJ} \cdot \mathrm{kmol}^{-1} \\
\mathrm{~kJ} \cdot \mathrm{kmol}^{-1} \\
\mathrm{~kJ} \cdot \mathrm{kmol}^{-1}\end{array}$ \\
\hline \multicolumn{2}{|l|}{$\begin{aligned} n_{1}=n_{-1} & =1.000 \times 10^{0} \\
n_{2}=n_{3} & =3.000 \times 10^{0} \\
n_{4} & =1.000 \times 10^{0} \\
n_{d} & =2.000 \times 10^{0}\end{aligned}$} \\
\hline $\begin{aligned} \tau & =3.000 \times 10^{2} \\
V & =1.000 \times 10^{-2} \\
\rho & =1.000 \times 10^{3} \\
c & =4.200 \times 10^{0} \\
T_{i} & =2.952 \times 10^{2}\end{aligned}$ & $\begin{array}{l}\mathrm{s} \\
\mathrm{m}^{3} \\
\mathrm{~kg} \cdot \mathrm{m}^{-3} \\
\mathrm{~kJ} \cdot \mathrm{kg}^{-1} \cdot \mathrm{K}^{-1} \\
\mathrm{~K}\end{array}$ \\
\hline
\end{tabular}

Table 1. Parameters of the CSTR Model

The control problem is to maintain the concentration $C_{A}$ and the temperature $T$ at the point $\left(C_{A}, T\right)=(1,400)$, where the selectivity $\mathcal{S}$ is maximum (i.e., $S=1$ ), in the presence of the process disturbances and model errors. The manipulated inputs are the inlet concentration of the reactant $\left(C_{A_{i}}\right)$ and the heat input to the reactor $(Q)$.

For time-discretization of the preceding continuous-time model, a simple forward difference method (Euler's) is used, which can provide a good approximate discrete-time model under fast sampling. The resulting discrete-time model is

$$
\left\{\begin{aligned}
{\left[\begin{array}{c}
C_{A}(k+1) \\
C_{U_{1}}(k+1) \\
T(k+1)
\end{array}\right] } & =\left[\begin{array}{c}
C_{A}(k)+f_{1}\left(C_{A}(k), C_{U_{1}}(k), T(k)\right) \Delta t+\frac{\Delta t}{\tau} u_{1}(k) \\
C_{U_{1}}(k)+f_{2}\left(C_{A}(k), C_{U_{1}}(k), T(k)\right) \Delta t \\
T(k)+f_{3}\left(C_{A}(k), C_{U_{1}}(k), T(k)\right) \Delta t+\frac{\Delta t}{\rho c_{p} V} u_{2}(k)
\end{array}\right] \\
y(k) & =\left[\begin{array}{c}
C_{A}(k) \\
T(k)
\end{array}\right],
\end{aligned}\right.
$$

The parameters of the reactor model are given in Table 1.

Figure 3 depicts the reaction selectivity $\mathbb{S}$, defined by

$$
\boldsymbol{S}=\frac{k_{d} C_{A}^{n_{d}}}{k_{1} C_{A}^{n_{1}}+k_{2} C_{A}^{n_{2}}+k_{3} C_{A}^{n_{3}}+k_{4} C_{A}^{n_{4}}-k_{-1} C_{U_{1}^{-1}}^{n_{1}}}
$$

vs. the concentration $C_{A}$ and temperature $T$ at steady-state conditions. As Figure 3 shows, the selectivity $\mathcal{S}$ has a global maximum at $\left(C_{A}, T\right)=(1,400)$. where $\Delta t$ is the sampling period, which is in the state-space form of Eq. 1. Here the vector of state variables $x=\left[C_{A} C_{U_{1}}\right.$ $T]^{T}$. The discrete-time model of Eq. 40 is employed to synthesize the controllers of Theorems 1,2 and 3 .

\section{Controller synthesis and implementation}

The first step is to calculate the relative orders and the characteristic matrix, according to Definitions 1 and 2. Since, 


$$
\begin{gathered}
\frac{\partial h_{1}(x)}{\partial x}=\left[\begin{array}{lll}
1 & 0 & 0
\end{array}\right], \quad \frac{\partial h_{2}(x)}{\partial x}=\left[\begin{array}{lll}
0 & 0 & 1
\end{array}\right], \\
\frac{\partial \Phi(x, u)}{\partial u}=\left[\begin{array}{cc}
\frac{\Delta t}{\tau} & 0 \\
0 & 0 \\
0 & \frac{\Delta t}{\rho c_{p} V}
\end{array}\right],
\end{gathered}
$$

and therefore,

$$
\begin{aligned}
& \frac{\partial h_{1}(x)}{\partial x} \frac{\partial \Phi(x, u)}{\partial u}=\frac{\Delta t}{\tau} \neq 0, \\
& \frac{\partial h_{2}(x)}{\partial x} \frac{\partial \Phi(x, u)}{\partial u}=\frac{\Delta t}{\rho c_{p} V} \neq 0,
\end{aligned}
$$

the relative orders $r_{1}=1$ and $r_{2}=1$, and the characteristic matrix is

$$
\mathcal{C}(x, u)=\left[\begin{array}{cc}
\frac{\Delta t}{\tau} & 0 \\
0 & \frac{\Delta t}{\rho c_{p} V}
\end{array}\right],
$$

which is nonsingular everywhere on $\mathbb{R}^{3} \times \mathbb{R}^{2}$.

For this specific example, the controllers of Theorems 1, 2, and 3 take the following forms:

(A) "Basic" GLC (All States Measurable). The controller of Theorem 1 (Eq. 24) becomes
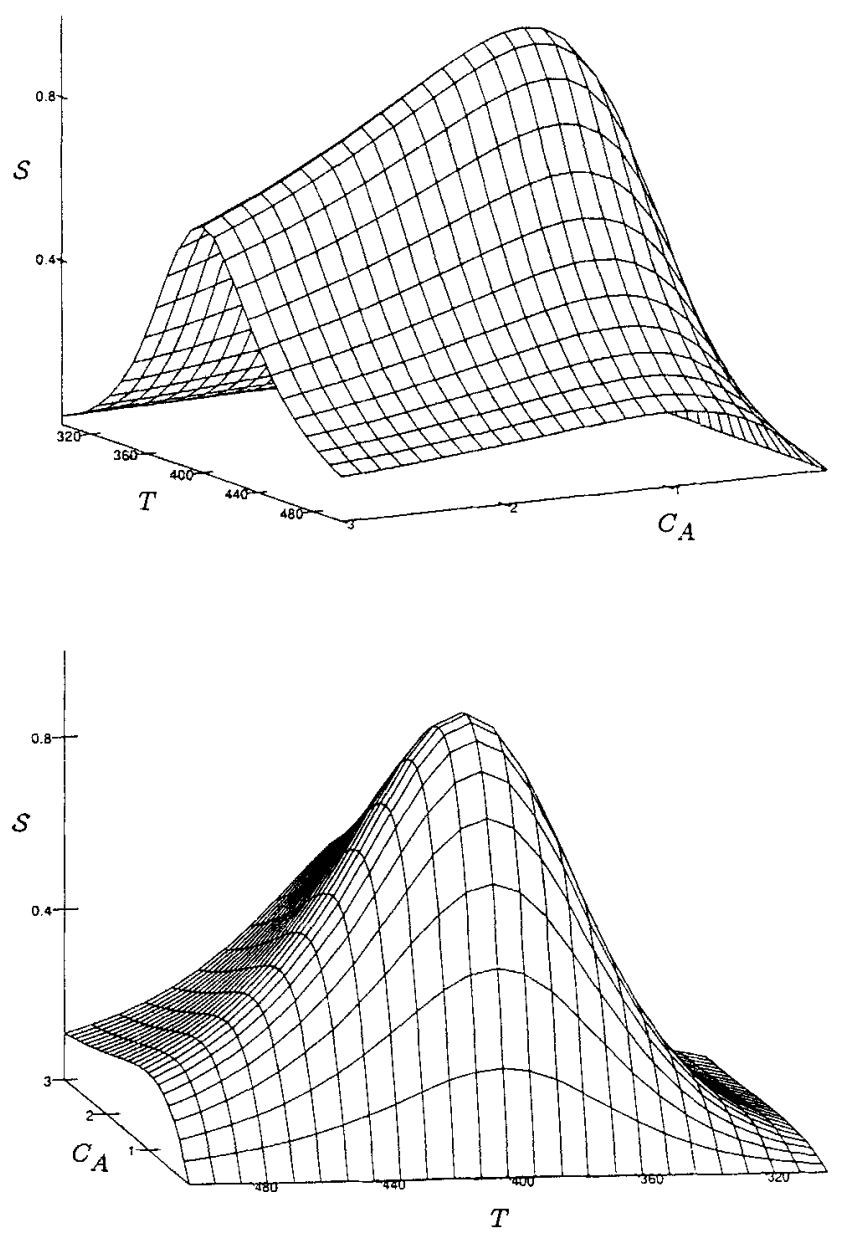

Figure 3. Reaction selectivity vs. $C_{A}$ and $T$, at steadystate conditions.

$\left\{\begin{aligned} \xi_{1}(k+1) & =\xi_{1}(k)+\left(1+\gamma_{11}^{1}\right) e_{1}(k) \\ \xi_{2}(k+1) & =\xi_{2}(k)+\left(1+\gamma_{21}^{2}\right) e_{2}(k) \\ u_{1}(k) & =\left\{\left(1+\beta_{11}^{1}\right)\left[\xi_{1}(k)-C_{A}(k)\right]+\left(1+\gamma_{11}^{1}\right) e_{1}(k)-f_{1}\left[C_{A}(k), C_{U_{1}}(k), T(k)\right] \Delta t\right\} \frac{\tau}{\Delta t} \\ u_{2}(k) & =\left\{\left(1+\beta_{21}^{2}\right)\left[\xi_{2}(k)-T(k)\right]+\left(1+\gamma_{21}^{2}\right) e_{2}(k)-f_{3}\left[C_{A}(k), C_{U_{1}}(k), T(k)\right] \Delta t\right\} \frac{\rho c_{p} V}{\Delta t}\end{aligned}\right.$

with the nominal initial conditions: $\xi_{1}(0)=C_{A}(0), \xi_{2}(0)=$ $T(0)$.

(B) Error-Feedback GLC. The controller of Theorem 2 (Eq. 26) becomes with the nominal initial conditions (see Table 2): $\xi_{1}(0)=$ $C_{A}(0), \xi_{2}(0)=T(0), \quad w_{1}(0)=C_{A}(0), \quad w_{2}(0)=C_{U}(0), w_{3}(0)=$ $T(0)$. Under the nominal initial conditions, $\xi_{1}(0)=w_{1}(0)$ and

$$
\left\{\begin{aligned}
\xi_{1}(k+1) & =\xi_{1}(k)+\left(1+\gamma_{11}^{1}\right) e_{1}(k) \\
\xi_{2}(k+1) & =\xi_{2}(k)+\left(1+\gamma_{21}^{2}\right) e_{2}(k) \\
w_{1}(k+1) & =w_{1}(k)+\left(1+\beta_{11}^{1}\right)\left(\xi_{1}(k)-w_{1}(k)\right)+\left(1+\gamma_{11}^{1}\right) e_{1}(k) \\
w_{2}(k+1) & =w_{2}(k)+f_{2}\left(w_{1}(k), w_{2}(k), w_{3}(k)\right) \Delta t \\
w_{3}(k+1) & =w_{3}(k)+\left(1+\beta_{21}^{2}\right)\left(\xi_{2}(k)-w_{3}(k)\right)+\left(1+\gamma_{21}^{2}\right) e_{2}(k) \\
u_{1}(k) & =\left\{\left(1+\beta_{11}^{1}\right)\left[\xi_{1}(k)-w_{1}(k)\right]+\left(1+\gamma_{11}^{1}\right) e_{1}(k)-f_{1}\left[w_{1}(k), w_{2}(k), w_{3}(k)\right] \Delta t\right\} \frac{\tau}{\Delta t} \\
u_{2}(k) & =\left\{\left(1+\beta_{21}^{2}\right)\left[\xi_{2}(k)-w_{3}(k)\right]+\left(1+\gamma_{21}^{2}\right) e_{2}(k)-f_{3}\left[w_{1}(k), w_{2}(k), w_{3}(k)\right] \Delta t\right\} \frac{\rho c_{p} V}{\Delta t}
\end{aligned}\right.
$$


Table 2. Operating Conditions

$\begin{array}{rlrl}C_{A}(0) & =0.000 \times 10^{9} & \mathrm{kmol} \cdot \mathrm{m}^{-3} \\ C_{U_{i}}(0) & =0.000 \times 10^{0} & \mathrm{kmol} \cdot \mathrm{m}^{-3} \\ T(0) & =2.952 \times 10^{2} & \mathrm{~K} \\ C_{A_{s s}} & =1.000 \times 10^{0} & \mathrm{kmol} \cdot \mathrm{m}^{-3} \\ C_{U_{1 s s}} & =5.844 \times 10^{-1} & \mathrm{kmol} \cdot \mathrm{m}^{-3} \\ T_{s s} & =4.000 \times 10^{2} & \mathrm{~K} \\ C_{A_{i s s}} & =5.687 \times 10^{0} & \mathrm{kmol} \cdot \mathrm{m}^{-3} \\ Q_{s s}^{i_{s s}} & =5.942 \times 10^{0} & \mathrm{~kJ} \cdot \mathrm{s}^{-1}\end{array}$

$\xi_{2}(0)=w_{3}(0)$, the controller of Eq. 42 has two redundant states: $\xi_{1}(k)=w_{1}(k), \xi_{2}(k)=w_{3}(k)$, for all $k$. Elimination of the two redundant states leads to the reduced-order realization of Corollary 1 (Eq. 27):

$$
\left\{\begin{aligned}
w_{1}(k+1) & =w_{1}(k)+\left(1+\gamma_{11}^{1}\right) e_{1}(k) \\
w_{2}(k+1) & =w_{2}(k)+f_{2}\left(w_{1}(k), w_{2}(k), w_{3}(k)\right) \Delta t \\
w_{3}(k+1) & =w_{3}(k)+\left(1+\gamma_{21}^{2}\right) e_{2}(k) \\
u_{1}(k) & =\left\{\left(1+\gamma_{11}^{1}\right) e_{1}(k)-f_{1}\left[w_{1}(k), w_{2}(k), w_{3}(k)\right] \Delta t\right\} \frac{\tau}{\Delta t} \\
u_{2}(k) & =\left\{\left(1+\gamma_{21}^{2}\right) e_{2}(k)-f_{3}\left[w_{1}(k), w_{2}(k), w_{3}(k)\right] \Delta t\right\} \frac{\rho c_{p} V}{\Delta t} .
\end{aligned}\right.
$$

(C) Mixed Error- and Output-Feedback GLC. Assuming that there is no secondary output $(s=0)$, the controller of Theorem 3 (Eq. 34) takes the form (note that the system of Eq. 40 is already in the form of Eq. 32): $\left(1+\gamma_{i 1}^{i}\right) / \Delta t$. Using a different strategy, each controller tries to induce the same decoupled input-output response of Eq. 45.

(ii) In the derivation of the controller of Eq. 42, an openloop observer was used: $w_{1}, w_{2}$, and $w_{3}$ represent the estimates of the concentration $C_{A}$, concentration $C_{U_{t}}$, and temperature $T$, respectively, obtained via openloop simulation of the process model. Under $\xi_{1}(0)=$ $w_{1}(0)$ and $\xi_{2}(0)=w_{3}(0)$, we will have $\xi_{1}(k)=w_{1}(k)$ and $\xi_{2}(k)=w_{3}(k)$ for all $k$. Therefore, in this case, the controller has two redundant modes. The elimination of the equations of the redundant states $\xi_{1}$ and $\xi_{2}$ yields the reduced-order controller of Eq. 43.

(iii) Controller (A) uses an on-line measurement of the concentration of $U_{1}$, whereas controllers (B) and (C)

$$
\left\{\begin{aligned}
\xi_{1}(k+1) & =\xi_{1}(k)+\left(1+\gamma_{11}^{1}\right) e_{1}(k) \\
\xi_{2}(k+1) & =\xi_{2}(k)+\left(1+\gamma_{21}^{2}\right) e_{2}(k) \\
\eta(k+1) & =\eta(k)+f_{2}\left(y_{1}(k), \eta(k), y_{2}(k)\right) \Delta t \\
u_{1}(k) & =\left\{\left(1+\beta_{11}^{1}\right)\left[\xi_{1}(k)-y_{1}(k)\right]+\left(1+\gamma_{11}^{1}\right) e_{1}(k)-f_{1}\left[y_{1}(k), \eta(k), y_{2}(k)\right] \Delta t\right\} \frac{\tau}{\Delta t} \\
u_{2}(k) & =\left\{\left(1+\beta_{21}^{2}\right)\left[\xi_{2}(k)-y_{2}(k)\right]+\left(1+\gamma_{21}^{2}\right) e_{2}(k)-f_{3}\left[y_{1}(k), \eta(k), y_{2}(k)\right] \Delta t\right\} \frac{\rho c_{p} V}{\Delta t}
\end{aligned}\right.
$$

with the nominal initial conditions (see Table 2): $\xi_{1}(0)=$ $C_{A}(0), \xi_{2}(0)=T(0), \eta(0)=C_{U_{1}}(0)$.

The tunable parameters of the controllers are chosen to be $\beta_{11}^{1}=\gamma_{11}^{1}=-0.90, \beta_{21}^{2}=\gamma_{21}^{2}=-0.96$, and $\beta_{11}^{2}=\gamma_{11}^{2}=\beta_{21}^{1}=$ $\gamma_{21}^{1}=0$ in all the cases. Under these settings, the three controllers try to induce the same decoupled linear input-output response

$$
\begin{aligned}
& y_{1}(k+1)+\gamma_{11}^{1} y_{1}(k)=\left(1+\gamma_{11}^{1}\right) y_{s p_{1}}(k) \\
& y_{2}(k+1)+\gamma_{21}^{2} y_{2}(k)=\left(1+\gamma_{21}^{2}\right) y_{s p_{2}}(k)
\end{aligned}
$$

to the overall closed-loop system.

Remark 1 . Some comments on the nature of the preceding controllers:

(i) All three controllers possess integral action: the state $\xi_{i}$ represents the integral of the error $e_{i}$ multiplied by temperature $T$ and the concentration $C_{A}$. In controller (B) (Eq. 42), the $w_{2}$-equation is exactly the $U_{1}$ balance equation and is driven by $w_{1}$ and $w_{3}$ rather than the on-line measurements of the concentration $C_{A}$ and reactor temperature $T$. Although $w_{1}$ and $w_{3}$ are supposed to match the concentration $C_{A}$ and the reactor temperature $T$ in closed-loop, disturbances and model errors will create a mismatch, which will impact upon the quality of the concentration estimate.

\section{Simulation results}

To simulate the reactor process, the standard software package ODEPACK is used to integrate numerically the three ordinary differential equations in $\mathrm{Eq}$. 39. Every $2.5 \mathrm{~s}$ (the sampling period $\Delta t$ ), the value(s) of $C_{A}, C_{U_{1}}$ and/or $T$ calculated by the ODE solver are used in the discrete-time con- 
Table 3. Simulation Cases

\begin{tabular}{lccc}
\hline \multicolumn{1}{c}{ GLC Structure } & $\begin{array}{c}\text { Nominal } \\
\text { Model }\end{array}$ & $\begin{array}{c}\text { Model } \\
\text { Error }\end{array}$ & $\begin{array}{c}\text { Initialization } \\
\text { Error }\end{array}$ \\
\hline "Basic" & A1 & A2 & - \\
Error-Feedback & B1 & B2 & B3 \\
Mixed Error- and Output-Feedback & C1 & C2 & C3 \\
\hline
\end{tabular}

trollers as the on-line process measurements. The operating conditions are given in Table 2.

The objective is to study the performance of the three controllers (A), (B), and (C) in (a) performing a smooth and fast reactor startup, and (b) rejecting the effect of a step change (20 K increase) in the inlet temperature $T_{i}$, under (1) perfect model (nominal case), (2) model errors (50\% error in the frequency factors of all the reactions), and (3) observer initialization errors $\left[w_{2}(0)=C_{U_{1}}(0)+2\right.$ for Case B3; and $\eta(0)=$ $C_{U}(0)+2$ for Case C3]. A list of the simulation cases is provided in Table 3.

Startup Performance. Figure 4a depicts the startup profiles of the outputs for the different cases; they show the almost identical performance of the three controllers. The output response $C_{A}$ under the model errors has a small overshoot that is insignificant relative to the magnitude of these errors. Overall, this figure shows the robustness of the controllers to the model and observer initialization errors. The corresponding startup profiles of the manipulated inputs are depicted in Figure 4b.

Figure $4 \mathrm{c}$ compares the variations of the actual and estimated values of the state $C_{U_{1}}$ during the period of startup
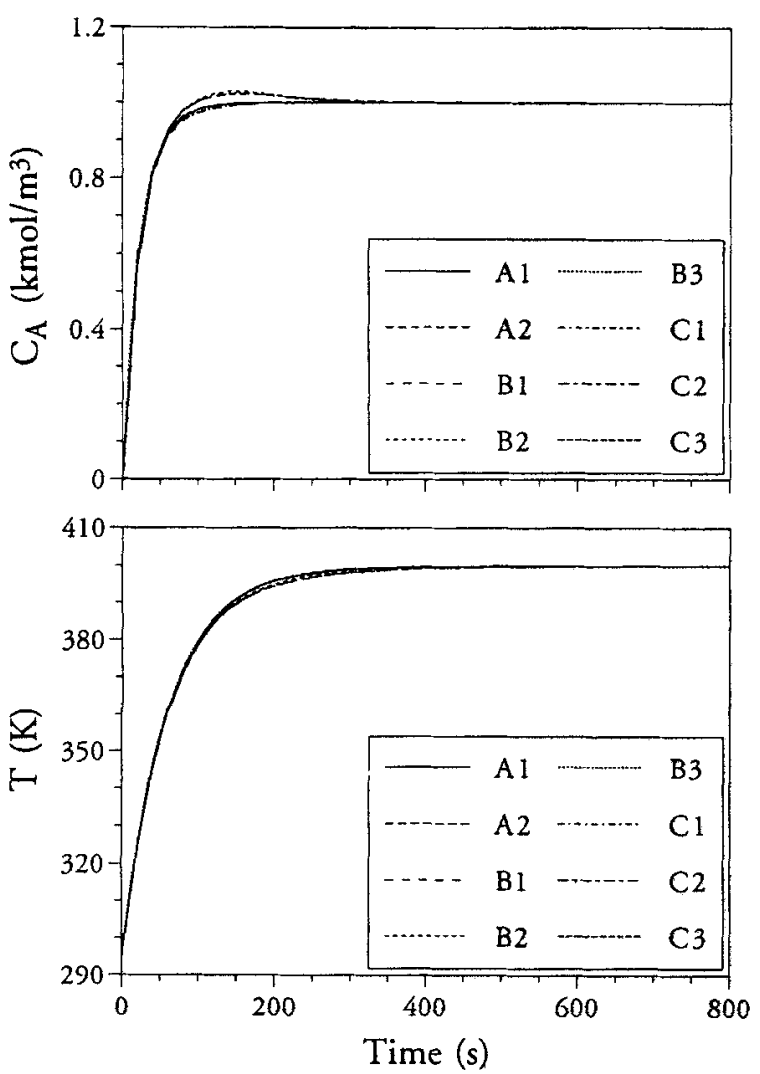

Figure 4a. Startup profiles of the controlled outputs.
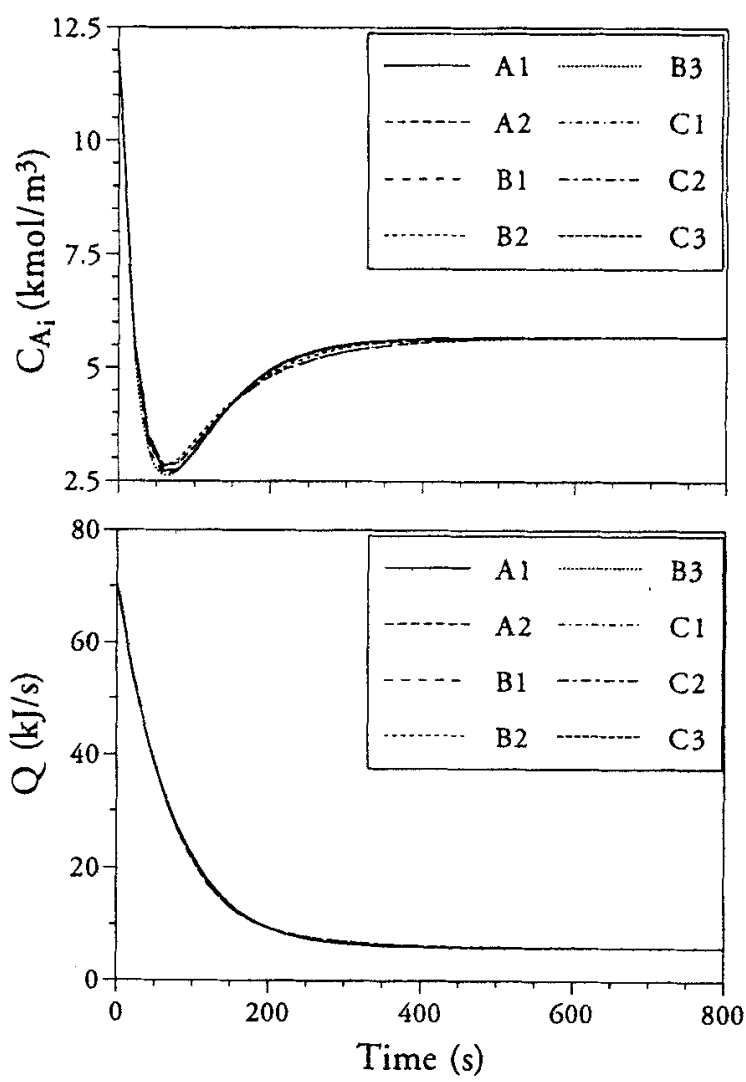

Figure 4b. Manipulated input profiles, corresponding to Figure 4a.
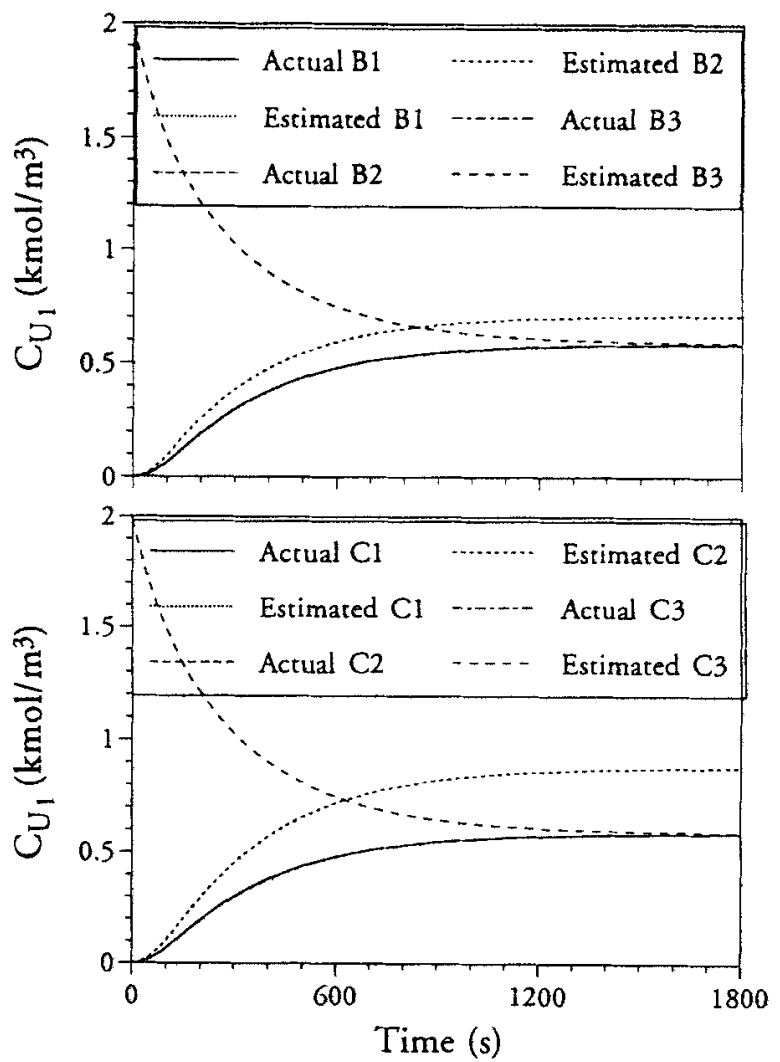

Figure 4c. Estimated and actual $C_{u_{1}}$ vs. time, corresponding to Figure $4 a$. 
(under controllers (B) and (C)): they show that, in the absence of the model errors, the observer initialization errors are rejected within less than $2,000 \mathrm{~s}$. However, in the presence of the model errors, the difference between the actual and estimated $C_{U_{1}}$ grows during the period of startup and is never rejected. Despite the mismatch between the estimated and actual $C_{U_{i}}$, the two controllers perform very satisfactorily in the presence of the model errors.

Regulatory Performance. Figure 5a (continuation of Figure $4 a$ ) depicts the performance of the controllers (under different conditions) in rejecting the effect of an unmeasurable step disturbance in $T_{i}$ (20 degrees increase, from $22^{\circ} \mathrm{C}$ to $42^{\circ} \mathrm{C}$, at $t=2,000 \mathrm{~s}$ ). The regulatory performance of controllers (A) and $(C)$ under both nominal conditions and model errors are almost identical. Controller (B), despite the larger initial errors, rejects asymptotically the effect of the disturbance on the outputs. This disturbance rejection is satisfactory relative to the magnitude of the applied disturbance. It should be noted that the output and the manipulated input profiles for Cases B3 and C3 (which are not shown here) are identical to those of Cases $\mathrm{B} 1$ and $\mathrm{C} 1$, respectively, since the initialization errors have already been rejected. The corresponding profiles of the manipulated inputs are depicted in Figure $5 b$ (which is the continuation of Figure $4 b$ ). This figure shows that the controller action in Cases $B 1$ and $B 2$ are initially less aggressive than those of controllers (A) and (B).

Figure $5 \mathrm{c}$ (continuation of Figure $4 \mathrm{c}$ ) compares the profiles of the actual and estimated values of the state $C_{U_{1}}$ when there is a step change ( $20 \mathrm{~K}$ increase) in the inlet temperature at
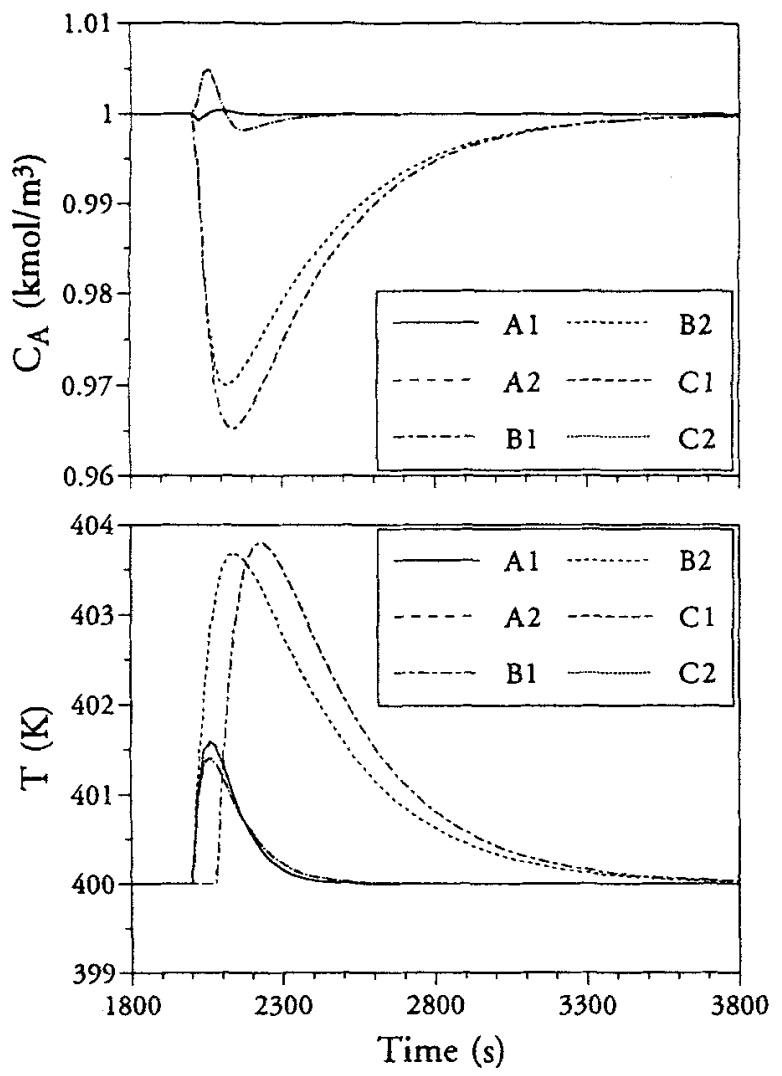

Figure 5a. Controlled-output profiles when there is a step change in $T_{i}$ at $t=2,000 \mathrm{~s}$.
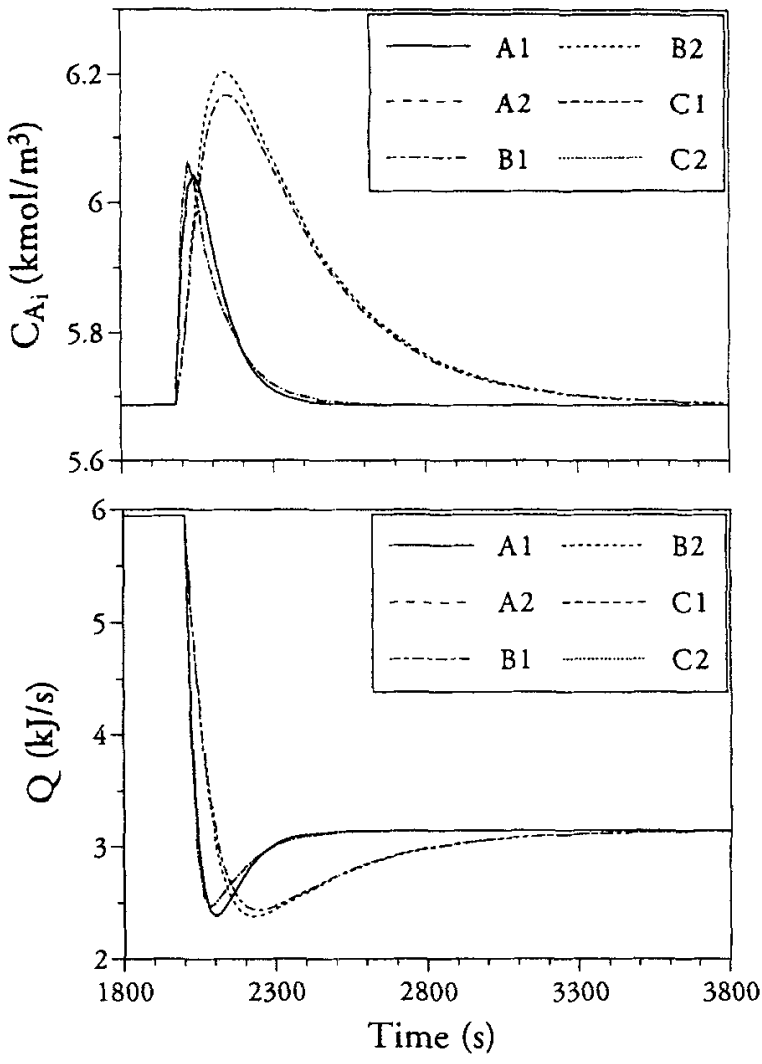

Figure 5b. Manipulated input profiles, corresponding to Figure 5a.
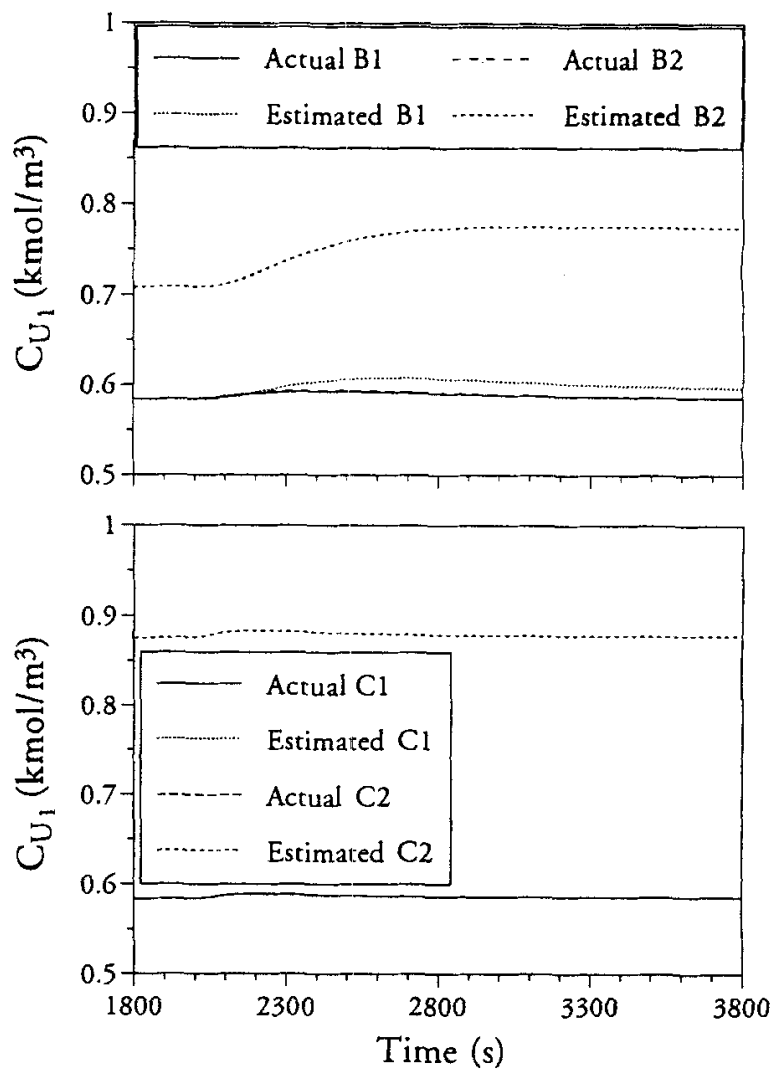

Figure 5c. Estimated and actual $\boldsymbol{C}_{U_{1}}$ vs. time, corresponding to Figure 5a. 
$t=2,000 \mathrm{~s}$ (corresponding to Figure 5a). In the presence of the model errors, when the inlet temperature disturbance is introduced at $t=2,000 \mathrm{~s}$, the difference between the actual and estimated $C_{U_{1}}$ grows more under controller (B). Under no model errors and controller $(C)$, the difference between the actual and estimated $C_{U_{1}}$ is zero and is not affected by the disturbance, whereas under controller (B), the difference grows as the disturbance is introduced.

\section{Conclusions}

Discrete-time nonlinear feedback control laws were derived for multivariable nonlinear processes whose delay-free parts are minimum phase, where a delay-free system is the one whose inverse is physically realizable. The derived controllers include mixed error- and state-feedback, error-feedback, and mixed error- and output-feedback laws, which can induce linear input-output closed-loop response. The mixed error- and output-feedback control law was derived for the broad class of the nonlinear processes, in which controlled outputs as well as some other process variables are measured. The conditions under which the mixed-error and output-feedback can be applied to a process operating at or around an open-loop unstable equilibrium point were determined. The application and performance of the derived control laws were illustrated by the numerical simulation of a chemical reactor.

Nonlinear control methods have been formulated both in continuous and discrete time. While the main advantage of the continuous-time formulation is that physical parameters are explicit in the process model and therefore in the control law, main advantages of discrete-time formulation are that (a) the process model and control law are directly suitable for computer implementation, and (b) the presence of deadtime does not complicate the control problem. Although several discrete-time nonlinear process model identification methods are available, at the present time there is no method to time-discretize exactly nonlinear continuous-time models. The mathematical models of many processes are obtained from the first principles, and therefore the resulting models are in continuous-time. There are a variety of approximate time-discretization methods, which are often referred to as numerical integration methods, such as Euler's method and the Runge-Kutta-Gill method. In the case that a continuoustime process model is available, one can use an approximate time-discretization method to obtain a discrete-time process model. This discrete-time process model can then be used to design a discrete-time feedback control law, for example, by using one of the synthesis formulas given in this article. In this case, the approximation in the time-discretization of the model can be viewed as a model error. Sufficient robustness with respect to the model error can be ensured by a proper tuning of the feedback controllers, as in the case of the reactor example considered in this article. Note that the approximate time-discretization issue also arises when we follow a continuous-time controller design approach, wherein a continuous-time controller is synthesized based on a continuous-time model, and then the controller has to be time-discretized before being implemented on a computer.

A nonlinear controller synthesis approach, in general, involves a state-feedback law (calculated on the basis of desirable closed-loop input-output response characteristics or by minimizing a performance index), and a state-observer to construct the states (for the case of incomplete state information). While there are a variety of ways to calculate a state feedback, at the present time there is no general method of nonlinear state-observer design. For the nonlinear process operating only within the domain of attraction of an openloop stable equilibrium point, one can use a full-order, open-loop observer to reconstruct the states. Open-loop observers can be applied to a broader class of nonlinear processes, if they are reduced-order observers driven by output measurements. The controller of Theorem 3 includes such an observer. In many chemical reactors, while the process dynamics possesses an open-loop unstable equilibrium point, the dynamics of the subsystem of the process that excludes temperature as a state variable has locally stable equilibrium points only. Because temperature is easily measurable, the controller of Theorem 3 is therefore applicable to a broad class of chemical reactors that exhibit steady-state multiplicities.

\section{Acknowledgment}

Financial support from the National Science Foundation through grant CTS-8912836 is gratefully acknowledged.

\section{Notation}

$c=$ heat capacity of reacting mixture, $\mathrm{kJ} \cdot \mathrm{kg}^{-1} \cdot \mathrm{K}^{-1}$

$C_{A}=$ concentration of reactant, $\mathrm{kmol} \cdot \mathrm{m}^{-3}$

$C_{A_{y s}}=$ steady-state concentration of reactant, $\mathrm{kmol} \cdot \mathrm{m}^{-3}$

$C_{A_{i}}=$ inlet concentration of reactant, $\mathrm{kmol} \cdot \mathrm{m}^{-3}$

$C_{A_{i}}=$ steady-state inlet concentration of reactant, $\mathrm{kmol} \cdot \mathrm{m}^{-3}$

$\stackrel{A_{i}}{C}=$ concentration of desired product, $\mathrm{kmol} \cdot \mathrm{m}^{-3}$

$C_{D}(0)=$ startup concentration of desired product, $\mathrm{kmol} \cdot \mathrm{m}^{-3}$

$C_{U_{\ell}}=$ concentration of undesired product $U_{\ell}, \mathrm{kmol} \cdot \mathrm{m}^{-3}$

$C_{U_{\ell}}(0)=$ startup concentration of undesired product $U_{\ell}, \mathrm{kmol} \cdot \mathrm{m}^{-3}$

$E_{a_{d}}=$ activation energy for desired reaction, $\mathrm{kJ} \cdot \mathrm{kmol}^{-1}$

$E_{a,}=$ activation energy for undesired reaction, $\ell, \mathrm{kJ} \cdot \mathrm{kmol}^{-1}$

$k_{d}=$ reaction rate constant for desired reaction, $\mathrm{m}^{3} \mathrm{kmol}^{-1} \cdot \mathrm{s}^{-1}$

$k_{1}, k_{4}=$ reaction rate constants for reactions 1 and $4, \mathrm{~s}^{-1}$

$k_{2}, k_{3}=$ reaction rate constants for reactions 2 and $3, \mathrm{~m}^{6} \cdot \mathrm{kmol}^{-2} \cdot \mathrm{s}^{-1}$

$k_{-1}=$ reaction rate constant for reaction $-1, \mathrm{~s}^{-1}$

$n_{\ell}=$ order of the reaction $\ell$

$R=$ universal gas constant, $\mathrm{kJ} \cdot \mathrm{kmol}^{-1} \cdot \mathrm{K}^{-1}$

$R_{A}=$ rate of production of $A, \mathrm{kmol} \cdot \mathrm{m}^{-3} \cdot \mathrm{s}^{-1}$

$R_{U_{1}}=$ rate of production of $U_{1}, \mathrm{kmol} \cdot \mathrm{m}^{-3} \cdot \mathrm{s}^{-1}$

$R_{H}=$ overall rate of heat production by reactions, $\mathrm{kJ} \cdot \mathrm{kmol}^{-1}$

$Q=$ heat input to reactor, $\mathrm{kJ} \cdot \mathrm{s}^{-1}$

$Q_{s s}=$ steady-state value of heat input, $\mathrm{kJ} \cdot \mathrm{s}^{-1}$

$T_{s s}=$ steady-state value of temperature, $\mathrm{K}$

$T(0)=$ startup temperature, $\mathrm{K}$

$V=$ volume of the reacting mixture, $\mathrm{m}^{3}$

$Z_{d}=$ frequency factor for desired reaction, $\mathrm{m}^{3} \cdot \mathrm{kmol}^{-1} \cdot \mathrm{s}^{-1}$

$Z_{1}, Z_{4}=$ frequency factors for reactions 1 and $4, \mathrm{~s}^{-1}$

$Z_{2}, Z_{3}=$ frequency factors for reactions 2 and $3, \mathrm{~m}^{6} \cdot \mathrm{kmol}^{-2} \cdot \mathrm{s}^{-1}$

$Z_{-1}=$ frequency factor for reaction $1, \mathrm{~s}^{-1}$

\section{Greek letters}

$-\Delta H_{d}=$ heat of desired reaction, $\mathrm{kJ} \cdot \mathrm{kmol}^{-1}$

$-\Delta H_{\ell}=$ heat of undesired reaction $\ell, \mathrm{kJ} \cdot \mathrm{kmol}^{-1}$

$\Phi=$ state vector function

$\rho=$ density of reacting mixture, $\mathrm{kg} \cdot \mathrm{m}^{-3}$

$\Psi=$ state feedback

$\tau=\mathrm{CSTR}$ residence time

\section{Literature Cited}

Bequette, B. W., “Nonlinear Control of Chemical Processes: A Review," Ind. Eng. Chem. Res., 30, 1391 (1991). 
Biegler, L. T., and J. B. Rawlings, "Optimization Approaches to Nonlinear Model Predictive Control," CPC IV , 543 (1991).

Byrnes, C. I., and A. Isidori, "Global Feedback Stabilization of Nonlinear Systems," Proc. IEEE CDC, Ft. Lauderdale, FL, p. 1031 (1985).

Chen, C. T., Linear System Theory and Design, Holt, Rinehart and Winston, New York (1984).

Daoutidis, P., and C. Kravaris, "Dynamic Output Feedback Control of Minimum-phase Nonlinear Processes," Chem. Eng. Sci., 49, 433 (1994).

Daoutidis, P., M. Soroush, and C. Kravaris, "Dynamic Output Feedback Control of Multivariable Nonlinear Processes," AIChE Meeting, Los Angeles (1991).

Economou, C. G., "An Operator Theory Approach to Nonlinear Controller Design," PhD Thesis, Cal. Inst. of Technol., Pasadena (1985).

Garcia, E. C., and M. Morari, "Internal Model Control: 3. Multivariable Control Law Computation and Tuning Guidelines," Ind. Eng. Chem., 24, 484 (1985a).

Garcia, E. C., and M. Morari, "Internal Model Control: 2. Design Procedure for Multivariable Systems," Ind. Eng. Chem., 24, 472 (1985b).

Glad, S. T., "Output Dead-Beat Control for Nonlinear Systems with One Zero at Infinity," Syst. Cont. Lett., 9, 249 (1987).

Hernandez, E., and Y. Arkun, "Control of Nonlinear Systems Using Polynomial ARMA Models," AIChE J., 39(3), 446 (1993).

Li, W. C., L. T. Biegler, C. G. Economou, and M. Morari, "A Constrained Pseudo-Newton Control Strategy for Nonlinear Systems," Comput. Chem. Eng., 14, 451 (1990).

Kravaris, C., and Y. Arkun, "Geometric Nonlinear Control-An Overview," CPC IV, 479 (1991).

Kravaris, C., and M. Soroush, "Synthesis of Multivariable Nonlinear Controllers by Input/Output Linearization," AIChE J., 36, 249 (1990).

Kravaris, C., P. Daoutidis, and R. Wright, "Output Feedback Control of Non-Minimum-Phase Nonlinear Processes," Chem. Eng. Sci., 29, 2107 (1994).

\section{Appendix: State-Space Realization of the External Controller}

A minimal-order state-space realization of the external controller is as follows:

$$
\begin{aligned}
\xi_{1}^{(1)}(k+1)=\xi_{r_{1}}^{(1)}(k)-\sum_{i=1}^{m} \sum_{\ell=1}^{r_{i}-1} \gamma_{i \ell}^{1}\left[\xi_{\ell}^{(i)}(k)-\xi_{r_{i}}^{(i)}(k)\right] & \\
& \quad+e_{1}(k)+\sum_{i=1}^{m} \sum_{\ell=1}^{r_{i}} \gamma_{\ell}^{1} e_{i}(k) \\
\xi_{2}^{(1)}(k+1)=\xi_{1}^{(1)}(k) & \\
\vdots & \\
\xi_{r_{1}}^{(1)}(k+1)= & \xi_{r_{1}-1}^{(1)}(k) \\
\vdots & \\
\xi_{1}^{(m)}(k+1)= & \xi_{r_{m}}^{(m)}(k)-\sum_{i=1}^{m} \sum_{\ell=1}^{r_{i}-1} \gamma_{i \ell}^{m}\left[\xi_{\ell}^{(i)}(k)-\xi_{r_{i}}^{(i)}(k)\right] \\
\xi_{2}^{(m)}(k+1)=\xi_{1}^{(m)}(k) & +e_{m}(k)+\sum_{i=1}^{m} \sum_{\ell=1}^{r_{i}} \gamma_{i \ell}^{m} e_{i}(k) \\
\vdots & \\
\xi_{r_{m}}^{(m)}(k+1)=\xi_{r_{1}-1}^{(m)}(k) &
\end{aligned}
$$

$$
\mathbb{B}_{0} v(k)=\left[\begin{array}{c}
\sum_{i=1}^{m}\left\{\sum_{\ell=1}^{r_{i}} \beta_{i \ell}{ }^{1} \xi_{\ell}^{(i)}(k)-\sum_{\ell=1}^{r_{i}-1} \gamma_{i \ell}^{1}\left[\xi_{\ell}^{(i)}(k)-\xi_{r_{i}}^{(i)}(k)\right]\right\}+\xi_{r_{1}}^{(1)}(k)+e_{1}(k)+\sum_{i=1}^{m} \sum_{\ell=1}^{r_{i}} \gamma_{i \ell}^{1} e_{i}(k) \\
\vdots \\
\sum_{i=1}^{m}\left\{\sum_{\ell=1}^{r_{i}} \beta_{i \ell}^{m} \xi_{\ell}^{(i)}(k)-\sum_{\ell=1}^{r_{i}-1} \gamma_{i \ell}^{m}\left[\xi_{\ell}^{(i)}(k)-\xi_{r_{i}}^{(i)}(k)\right]\right\}+\xi_{r_{m}}^{(m)}(k)+e_{m}(k)+\sum_{i=1}^{m} \sum_{\ell=1}^{r_{i}} \gamma_{i \ell}^{m} e_{i}(k)
\end{array}\right]
$$

McLellan, P. J., T. J. Harris, and D. W. Bacon, "Error Trajectory Descriptions of Nonlinear Controller Designs," Chem. Eng. Sci., 45, 3017 (1990).

Mehra, R. K., and R. Rouhani, "Theoretical Considerations on Model Algorithmic Control for Nonminimum Phase Systems," Proc. $A C C$, TA8-B (1980).

Monaco, S., and D. Normand-Cyrot, "Zero Dynamics of Sampled Nonlinear Systems,” Syst. Cont. Lett., 11, 229 (1988).

Nijmeijer, H., and A. J. van der Schaft, Nonlinear Dynamical Control Systems, Springer-Verlag, New York (1990).

O'Reilly, J., "The Discrete Linear Time Invariant Time-Optimal Control Problem-An Overview," Automatica, 17, 363 (1981).

Richalet, J., A. Rault, J. L. Testud, and J. Papon, "Model Predictive Heuristic Control: Application to Industrial Processes," Automatica, 14, 413 (1978).

Soroush, M., "Studies in Nonlinear Control and Optimal Design with Experimental Application to Polymerization Reactors," PhD Thesis, Univ. of Michigan, Ann Arbor (1992).

Soroush, M., and C. Kravaris, "A Continuous-Time Formulation of Nonlinear Model Predictive Control," Proc. ACC., 1561 (1992a).

Soroush, M., and C. Kravaris, "Discrete-Time Nonlinear Controller Synthesis by Input/Output Linearization," AIChE J., 38, 1923 (1992b). or, in a more compact form,

$$
\begin{gathered}
\xi(k+1)=A_{e} \xi(k)+B_{e} e(k) \\
\mathfrak{Q}_{0} v(k)=C_{e} \xi(k)+D_{e} e(k),
\end{gathered}
$$

where $\xi(k)=\left[\xi_{1}^{(1)}(k) \cdots \xi_{r_{1}}^{(1)}(k): \cdots: \xi_{1}^{(m)}(k) \cdots \xi_{r_{m}}^{(m)}(k)\right]^{T}$, and $A_{e}, B_{e}, C_{e}$, and $D_{e}$ are the appropriate $\left(r_{1}+\cdots+r_{m}\right) \times\left(r_{1}\right.$ $\left.+\cdots+r_{m}\right),\left(r_{1}+\cdots+r_{m}\right) \times m, m \times\left(r_{1}+\cdots+r_{m}\right)$, and $m \times$ $m$ matrices arising from the state-space realization of Eq. A1.

Manuscript received May 23, 1994, and revision received Jan. 9, 1995. 